\title{
THE MOMENT MAP ON SYMPLECTIC VECTOR SPACE AND OSCILLATOR REPRESENTATION
}

\author{
TAKASHI HASHIMOTO
}

\begin{abstract}
Let $G$ denote $\operatorname{Sp}(n, \mathbb{R}), \mathrm{U}(p, q)$ or $\mathrm{O}^{*}(2 n)$. The main aim of this paper is to show that the canonical quantization of the moment map on a symplectic $G$-vector space $(W, \omega)$ naturally gives rise to the oscillator (or Segal-Shale-Weil) representation of $\mathfrak{g}:=\mathrm{Lie}(G) \otimes \mathbb{C}$. More precisely, after taking a complex Lagrangian subspace $V$ of the complexification of $W$, we assign an element of the Weyl algebra for $V$ to $\langle\mu, X\rangle$ for each $X \in \mathfrak{g}$, which we denote by $\langle\widehat{\mu}, X\rangle$. Then we show that the map $X \mapsto \mathrm{i}\langle\widehat{\mu}, X\rangle$ gives a representation of $\mathfrak{g}$. With a suitable choice of $V$ in each case, the representation coincides with the oscillator representation of $\mathfrak{g}$.
\end{abstract}

\section{INTRODUCTION}

Let $(W, \omega)$ be a symplectic vector space and $\operatorname{Sp}(W)$ the group of linear symplectic isomorphisms of $W$. Then it is well known that each component of the moment map, i.e., the Hamiltonian function $H_{X}$ on $W$, is quadratic in the coordinate functions for any $X \in \mathfrak{s p}(W)$ (see e.g. [CG97]). Therefore, taking account of the fact that the commutators among the quantized operators corresponding to the coordinate functions are central, one can see that the canonical quantization gives a representation of $\mathfrak{s p}(W)$ since the map $X \mapsto H_{X}$ is a Lie algebra homomorphism from $\mathfrak{s p}(W)$ into $C^{\infty}(W)$, where the latter is regarded as a Lie algebra of infinite dimension with respect to the Poisson bracket.

The main aim of this paper is to show that for real reductive Lie groups $G=$ $\operatorname{Sp}(n, \mathbb{R}), \mathrm{U}(p, q)$ and $\mathrm{O}^{*}(2 n)$, the canonical quantization of the moment map on real symplectic $G$-vector space ( $W, \omega)$ gives rise to the oscillator (or Segal-Shale-Weil) representation of the complexified Lie algebra $\mathfrak{g}$ of $\mathfrak{g}_{0}:=\operatorname{Lie}(G)$ in a natural way. Here, we understand that the canonical quantization is to construct a mapping from the space of smooth functions on $W$ into the ring of polynomial coefficient differential operators on a complex Lagrangian subspace $V$ of the complexification $W_{\mathbb{C}}$ of $W$, the so-called Weyl algebra for $V$, that induces a Lie algebra homomorphism from $g$ into the Weyl algebra. We remark that a different choice of a Lagrangian subspace results in a different quantization, and hence a different representation of the Lie algebra. In fact, when $G=\mathrm{U}(p, q)$ and $\mathrm{O}^{*}(2 n)$, we will find in $\S \S 3-5$ that one choice of a Lagrangian subspace produces finite-dimensional irreducible representations of $\mathfrak{g}$, while another produces infinite-dimensional ones (i.e. the oscillator representations).

Date: July 22, 2018.

2010 Mathematics Subject Classification. Primary: 22E46, 17B20; Secondary: 81S10.

Key words and phrases. symplectic vector space, moment map, canonical quantization, oscillator representation, Howe duality .

Partly supported by JSPS Grant-in-Aid for Scientific Research (C) No. 23540203 and No. 26400014. 
The oscillator representations have been extensively studied in relation to the Howe duality and to the minimal representations. Note that each Lie group $G$ we consider in this paper is a counterpart of the Howe's reductive dual pair $\left(G, G^{\prime}\right)$ with $G^{\prime}$ compact, i.e., $G$ and $G^{\prime}$ are centralizer of each other in a symplectic group $\operatorname{Sp}(N, \mathbb{R})$ for some $N$. One can obtain the oscillator representations by embedding $G$ into $\operatorname{Sp}(N, \mathbb{R})$ for $G=\mathrm{U}(p, q)$ and $\mathrm{O}^{*}(2 n)$ (see [KV78, EHW83, How89a, How89b, How85, DES91] etc.).

As for another approach to a construction of the oscillator representations, we should mention [HKMØ12], in which they construct the oscillator representations via Jordan algebras when $G$ is an arbitrary Hermitian Lie group of tube type.

It was shown in [Has11] that for the classical Hermitian symmetric pairs $(G, K)=$ $(\mathrm{SU}(p, q), \mathrm{S}(\mathrm{U}(p) \times \mathrm{U}(q))),(\mathrm{Sp}(n, \mathbb{R}), \mathrm{U}(n))$, and $\left(\mathrm{SO}^{*}(2 n), \mathrm{U}(n)\right)$, one obtains generating functions of the principal symbols of $K_{\mathbb{C}}$-invariant differential operators on $G / K$ in terms of determinant or Pfaffian of a certain $\mathfrak{g}$-valued matrix whose entries are the total symbols of the differential operators corresponding to the holomorphic discrete series representations realized via Borel-Weil theory, where $K_{\mathbb{C}}$ denotes a complexification of $K$. We note that the $K_{\mathbb{C}}$-invariant differential operators play a prominent rôle in the Capelli identity (see [HU91]). Moreover, we also clarified in [Has11] that the g-valued matrix mentioned above can be regarded as the twisted moment map $\mu_{\lambda}$ on the cotangent bundle of $G / K$ which reduces to the moment map $\mu$ on the cotangent bundle when $\lambda \rightarrow 0$, where $\lambda$ is an element of $\mathfrak{g}^{*}$, the dual space of $\mathfrak{g}$, that parametrizes the representations. In summary, one can say that the moment map relates non-commutative objects (representation operators which are realized as differential operators) to commutative ones (symbols of the differential operators). Now in this paper, we will proceed in the reverse direction: from commutative objects to non-commutative ones.

In the remainder of this section, we review a few relevant notions from symplectic geometry briefly, and state our main result.

Let $(M, \omega)$ be a real symplectic manifold. For $f \in C^{\infty}(M)$, the space of smooth $\mathbb{R}$-valued functions on $M$, let $\xi_{f}$ denote the vector field on $M$ satisfying $\iota\left(\xi_{f}\right) \omega=\mathrm{d} f$, where $\iota$ stands for the contraction. Then we define the Poisson bracket by

$$
\{f, g\}:=\omega\left(\xi_{g}, \xi_{f}\right) \quad\left(f, g \in C^{\infty}(M)\right),
$$

which we extend to the space of smooth $\mathbb{C}$-valued functions by linearity. If we denote the quantum observable corresponding to a classical observable $f \in C^{\infty}(M)$ by $\widehat{f}$, then the quantization principles require in particular that

$$
\text { if }\left\{f_{1}, f_{2}\right\}=f_{3} \text { then }\left[\widehat{f_{1}}, \widehat{f_{2}}\right]=-\mathrm{i} \hbar \widehat{f_{3}}
$$

where $\hbar$ is the Planck constant (see e.g. [BW97, Woo91]); we set $\hbar=1$ for simplicity in what follows.

Suppose that a Lie group $G$ acts on $M$ symplectically, i.e., $g^{*} \omega=\omega$ for all $g \in G$. A smooth map $\mu: M \rightarrow \mathrm{g}_{0}^{*}$ is called the moment map if the following conditions hold: $\mu$ is $G$-equivariant, and it satisfies

$$
\mathrm{d}\langle\mu, X\rangle=\iota\left(X_{M}\right) \omega \quad \text { for all } X \in \mathfrak{g}_{0},
$$


where $\mathfrak{g}_{0}^{*}$ is the dual space of $\mathfrak{g}_{0}$ and $X_{M}$ denotes the vector field on $M$ defined by

$$
X_{M}(p)=\left.\frac{\mathrm{d}}{\mathrm{d} t}\right|_{t=0} \exp (-t X) \cdot p \quad(p \in M) .
$$

We often identify $\mathfrak{g}_{0}^{*}$ with $\mathfrak{g}_{0}$ via the nondegenerate symmetric invariant bilinear form $B$ defined by

$$
B(X, Y)=\left\{\begin{array}{lll}
\frac{1}{2} \operatorname{tr}(X Y) & \text { if } & \mathfrak{g}_{0}=\mathfrak{s p}(n, \mathbb{R}) \text { or } \mathfrak{b}^{*}(2 n) \\
\operatorname{tr}(X Y) & \text { if } & \mathfrak{g}_{0}=\mathfrak{u}(p, q),
\end{array}\right.
$$

which extends to the one on $\mathfrak{g}=\mathfrak{s p}_{n}, \mathfrak{o}_{2 n}$, or $\mathfrak{g l}_{p+q}$, the complexification of $\mathfrak{g}_{0}=\mathfrak{s p}(n, \mathbb{R})$, $\mathfrak{D}^{*}(2 n)$ or $\mathfrak{u}(p, q)$. If there is no risk of confusion, we denote the composition of $\mu$ and the isomorphism $\mathfrak{g}_{0}^{*} \simeq \mathfrak{g}_{0}$ also by $\mu$. Our symplectic $G$-manifold $(M, \omega)$ will be a real symplectic $G$-vector space.

The main result of this paper is the following, which we prove case by case:

Theorem. Let $G=\operatorname{Sp}(n, \mathbb{R}), \mathrm{U}(p, q)$ and $\mathrm{O}^{*}(2 n)$, and let $(W, \omega)$ be the real symplectic $G$-vector spaces $W=\mathbb{R}^{2 n},\left(\mathbb{C}^{p+q}\right)_{\mathbb{R}}$ and $\left(\mathbb{C}^{2 n}\right)_{\mathbb{R}}$ equipped with $\omega$ given by

$$
\omega(v, w)=\left\{\begin{array}{lll}
{ }^{t} v J_{n} w & \text { if } & W=\mathbb{R}^{2 n}, \\
\operatorname{Im}\left(v^{*} I_{p, q} w\right) & \text { if } & W=\left(\mathbb{C}^{p+q}\right)_{\mathbb{R}}, \\
\operatorname{Im}\left(v^{*} I_{n, n} w\right) & \text { if } & W=\left(\mathbb{C}^{2 n}\right)_{\mathbb{R}}
\end{array}\right.
$$

for $v, w \in W$, respectively, where $J_{n}=\left[\begin{array}{c}1_{n} \\ -1_{n}\end{array}\right]$ and $I_{p, q}=\left[\begin{array}{cc}1_{p} & \\ & -1_{q}\end{array}\right]$. Then, with certain choice of complex Lagrangian subspace of the complexification $W_{\mathbb{C}}$ of $W$, the canonical quantization of the moment map $\mu: W \rightarrow \mathrm{g}_{0}^{*}$ yields the oscillator representations.

The rest of this paper is organized as follows. In $\S 2$, we consider the case where $G=\operatorname{Sp}(n, \mathbb{R})$, which is the most fundamental in this paper in the sense that a choice of a complex Lagrangian subspace is the key to obtain the oscillator representation. The original motivation of this project has started from this case with $n=1$. In $\S 3$, we turn to the case where $G=\mathrm{U}(p, q)$ and show that the canonical quantization of the moment map with a certain choice of a complex Lagrangian subspace yields irreducible finite-dimensional representations of $\mathfrak{g l}_{p+q}$, and postpone showing that another choice leads to the oscillator representations of $\mathfrak{g l}_{p+q}$ until $\S 5$. In $\S 4$, we treat the case $G=\mathrm{O}^{*}(2 n)$, in which the moment map can be expressed in two ways due to the fact that the quaternionic vector space $\mathbb{H}^{n}$ is $\mathbb{C}$-isomorphic to $\mathbb{C}^{2 n}$ and to $\operatorname{Mat}_{n \times 2}(\mathbb{C})$. In $\S 5$, we take complex Lagrangian subspaces different from the ones considered in $\S \S 3$ and 4 in the cases of $\mathrm{U}(p, q)$ and $\mathrm{O}^{*}(2 n)$ : one leading to finite-dimensional irreducible representations when $\mathfrak{g}=\mathfrak{v}_{2 n}$, and one leading to the oscillator representation when $\mathfrak{g}=\mathfrak{g l}_{p+q}$. Finally, we remark a relation between the moment map and the associated variety of the corresponding irreducible $\mathfrak{g}$-modules occurring in the irreducible decomposition of the space of polynomials on the Lagrangian subspace under the joint action of the dual pairs $\left(\mathfrak{g}, G^{\prime}\right)$.

Notation: (i) Throughout the paper, we fix a Cartan involution $\theta$ to be given by $\theta X=$ $-X^{*}$. Let $\mathfrak{g}_{0}=\mathfrak{f}_{0} \oplus \mathfrak{p}_{0}$ denote the Cartan decomposition for $\mathfrak{g}_{0}$, and $\mathfrak{g}=\mathfrak{f} \oplus \mathfrak{p}$ the corresponding complexified Cartan decomposition for $\mathfrak{g}=\mathfrak{g}_{0} \otimes \mathbb{C}$. 
For a given basis $\left\{X_{\alpha}\right\}_{\alpha}$ for $\mathfrak{g}_{0}$ (resp. $\mathfrak{g}$ ), let us denote by $\left\{X_{\alpha}^{\vee}\right\}$ its dual basis with respect to $B$, i.e., the basis for $\mathfrak{g}_{0}$ (resp. $\mathfrak{g}$ ) satisfying

$$
B\left(X_{\alpha}, X_{\beta}^{\vee}\right)=\delta_{\alpha, \beta},
$$

where $\delta_{\alpha, \beta}$ is the Kronecker's delta, i.e., is equal to 1 if $\alpha=\beta$ and 0 otherwise.

(ii) For a positive integer $i$, we set

$$
\bar{\imath}:=\left\{\begin{array}{lll}
n+i & \text { if } & \mathfrak{g}=\mathfrak{s p}_{n} \text { or } \mathfrak{b}_{2 n} \\
p+i & \text { if } & \mathfrak{g}=\mathfrak{g l}_{p+q},
\end{array}\right.
$$

where $\mathfrak{s p}_{n}, \mathfrak{D}_{2 n}$ and $\mathfrak{g l}_{p+q}$ denote the complexified Lie algebras of $\mathfrak{s p}(n, \mathbb{R}), \mathfrak{o}^{*}(2 n)$ and $\mathfrak{u}(p, q)$ respectively.

\section{Reductive DUAL PAIR $\left(\mathfrak{s p}(n, \mathbb{R}), \mathrm{O}_{k}\right)$}

In this section, let $G$ denote the symplectic group $\operatorname{Sp}(n, \mathbb{R})$ of rank $n$ over $\mathbb{R}$ which we realize as

$$
\operatorname{Sp}(n, \mathbb{R})=\left\{g \in \mathrm{GL}_{2 n}(\mathbb{R}) ;{ }^{t} g J_{n} g=J_{n}\right\}
$$

with $J_{n}=\left[\begin{array}{c}1_{n} \\ { }_{-1}\end{array}\right]$. Set $\mathfrak{g}_{0}=\mathfrak{s p}(n, \mathbb{R})$, the Lie algebra of $G$, and take a basis for $\mathfrak{g}_{0}$ as follows:

$$
\begin{array}{ll}
X_{i, j}^{0}=E_{i, j}-E_{\bar{\jmath}, \bar{l}} & (1 \leqslant i, j \leqslant n), \\
X_{i, j}^{+}=E_{i, \bar{j}}+E_{j, \bar{l}} & (1 \leqslant i \leqslant j \leqslant n), \\
X_{i, j}^{-}=E_{\bar{l}, j}+E_{\bar{\jmath}, i} & (1 \leqslant i \leqslant j \leqslant n),
\end{array}
$$

where $E_{i, j}$ denotes the matrix unit of size $2 n \times 2 n$, i.e., its $(i, j)$-th entry is 1 and all other entries are 0 . Note that they also form a basis for $\mathfrak{g}=\mathfrak{s p}_{n}$.

2.1. Let $W=\mathbb{R}^{2 n}$ which is equipped with the canonical symplectic form $\omega$ given by

$$
\omega(v, w)={ }^{t} v J_{n} w \quad(v, w \in W) .
$$

Obviously, the natural left action of $G$ on $W$ defined by $v \mapsto g v$ (matrix multiplication) for $v \in W$ and $g \in G$ is symplectic, i.e., $g^{*} \omega=\omega$ for all $g \in G$. If we identify the canonical base vectors $e_{i}:={ }^{t}(0, \ldots, 0,1,0, \ldots, 0)$ with $\partial_{x_{i}}$ for $i=1,2, \ldots, n$ and with $\partial_{y_{i-n}}$ for $i=\overline{1}, \overline{2}, \ldots, \bar{n}$, then it is written as

$$
\omega=\sum_{i=1}^{n} \mathrm{~d} x_{i} \wedge \mathrm{d} y_{i}
$$

at $v={ }^{t}\left(x_{1}, \ldots, x_{n}, y_{1}, \ldots, y_{n}\right) \in W$.

Lemma 2.1. The vector fields on Wgenerated by the basis (2.1) for $\mathfrak{g}_{0}=\mathfrak{s p}(n, \mathbb{R})$ in the sense of (1.4) are given by

$$
\begin{array}{ll}
\left(X_{i, j}^{0}\right)_{W}=-x_{j} \partial_{x_{i}}+y_{i} \partial_{y_{j}} & (1 \leqslant i, j \leqslant n), \\
\left(X_{i, j}^{+}\right)_{W}=-\left(y_{j} \partial_{x_{i}}+y_{i} \partial_{x_{j}}\right) & (1 \leqslant i \leqslant j \leqslant n), \\
\left(X_{i, j}^{-}\right)_{W}=-\left(x_{j} \partial_{y_{i}}+x_{i} \partial_{y_{j}}\right) & (1 \leqslant i \leqslant j \leqslant n) .
\end{array}
$$

Proof. It is an easy exercise to show these formulae. 
Note that the orthogonal group $\mathrm{O}(1)=\{ \pm 1\}$ also acts on $W$ symplectically on the right.

Proposition 2.2. Let $(W, \omega)$ be as above and $G=\operatorname{Sp}(n, \mathbb{R})$. Then the moment map $\mu: W \rightarrow \mathfrak{g}_{0}^{*} \simeq \mathfrak{g}_{0}$ is given by

$$
\mu(v)=v^{t} v J_{n}=\left[\begin{array}{ll}
-x^{t} y & x^{t} x \\
-y^{t} y & y^{t} x
\end{array}\right]
$$

for $v={ }^{t}\left(x_{1}, \ldots, x_{n}, y_{1}, \ldots, y_{n}\right) \in W$ with $x={ }^{t}\left(x_{1}, \ldots, x_{n}\right)$ and $y={ }^{t}\left(y_{1}, \ldots, y_{n}\right)$. In particular, $\mu$ is G-equivariant and is $\mathrm{O}(1)$-invariant.

Proof. In order to make this paper self-contained, we include the proof (see, however, e.g. [CG97, Proposition 1.4.6]). It follows from Lemma 2.1 that

$$
\begin{aligned}
\mathrm{d}\left\langle\mu, X_{i, j}^{0}\right\rangle & =\iota\left(\left(X_{i, j}^{0}\right)_{W}\right) \omega \\
& =\iota\left(-x_{j} \partial_{x_{i}}+y_{i} \partial_{y_{j}}\right) \sum_{k=1}^{n} \mathrm{~d} x_{k} \wedge \mathrm{d} y_{k} \\
& =-x_{j} \mathrm{~d} y_{i}-y_{i} \mathrm{~d} x_{j}=-\mathrm{d}\left(y_{i} x_{j}\right) .
\end{aligned}
$$

Hence one obtains that

Similar calculations yield

$$
\left\langle\mu, X_{i, j}^{0}\right\rangle=-y_{i} x_{j}
$$

$$
\left\langle\mu, X_{i, j}^{+}\right\rangle=-y_{i} y_{j} \quad \text { and } \quad\left\langle\mu, X_{i, j}^{-}\right\rangle=x_{i} x_{j}
$$

Therefore,

$$
\begin{aligned}
\mu(v) & =\sum_{i, j}\left\langle\mu, X_{i, j}^{0}\right\rangle\left(X_{i, j}^{0}\right)^{\vee}+\sum_{i \leqslant j}\left\langle\mu, X_{i, j}^{+}\right\rangle\left(X_{i, j}^{+}\right)^{\vee}+\sum_{i \leqslant j}\left\langle\mu, X_{i, j}^{-}\right\rangle\left(X_{i, j}^{-}\right)^{\vee} \\
& =\sum_{i, j}\left(-y_{i} x_{j}\right)\left(E_{j, i}-E_{\bar{l}, \bar{j}}\right)+\sum_{i \leqslant j}\left(-y_{i} y_{j}\right) 2^{-\delta_{i j}}\left(E_{i, \bar{j}}+E_{j, \bar{l}}\right)+\sum_{i \leqslant j} x_{i} x_{j} 2^{-\delta_{i j}}\left(E_{\bar{l}, j}+E_{\bar{j}, i}\right) \\
& =\sum_{i, j}\left(-x_{i} y_{j} E_{i, j}+x_{i} x_{j} E_{i, \bar{j}}-y_{i} y_{j} E_{\bar{l}, j}+y_{i} x_{j} E_{\bar{l}, \bar{j}}\right) \\
& =\left[\begin{array}{ll}
-x^{t} y & x^{t} x \\
-y^{t} y & y^{t} x
\end{array}\right]=v^{t} v J_{n}
\end{aligned}
$$

for $v={ }^{t}\left(x_{1}, \ldots, y_{n}\right)$ with $x={ }^{t}\left(x_{1}, \ldots, x_{n}\right)$ and $y={ }^{t}\left(y_{1}, \ldots, y_{n}\right)$.

Now the $\mathrm{O}(1)$-invariance of $\mu$ is trivial, and the $G$-equivariance can be verified as follows:

$$
\mu(g v)=g v^{t}(g v) J_{n}=g v^{t} v^{t} g J_{n}=g v^{t} v J_{n} g^{-1}=\operatorname{Ad}(g) \mu(v)
$$

since ${ }^{t} g J_{n}=J_{n} g^{-1}$ for $g \in G$. This completes the proof.

It follows from the definitions of the Poisson bracket (1.1) and the symplectic form (2.3) that

$$
\left\{x_{i}, y_{j}\right\}=-\delta_{i, j}, \quad\left\{x_{i}, x_{j}\right\}=\left\{y_{i}, y_{j}\right\}=0
$$

for $i, j=1, \ldots, n$. In view of (2.6), we quantize the classical observables by assigning

$$
\widehat{x}_{i}=\text { multiplication by } x_{i}, \quad \widehat{y}_{i}=-\mathrm{i} \partial_{x_{i}},
$$


so that $\left[\widehat{x}_{i}, \widehat{y}_{j}\right]=\mathrm{i} \delta_{i, j}$, as required. In what follows, we simply denote the multiplication operator by a function $f$ by the same letter $f$ if there is no risk of confusion.

Note that the quantization (2.7) corresponds to taking a Lagrangian subspace of $W$ spanned by $e_{1}, \ldots, e_{n}$. However, in order to obtain a representation of the complex Lie algebra $\mathfrak{g}=\mathfrak{s p}_{n}$, we will take a complex Lagrangian subspace of the complexification $W_{\mathbb{C}}$ defined by

$$
V:=\left\langle e_{1}, \ldots, e_{n}\right\rangle_{\mathbb{C}}
$$

Therefore, the classical observables $x_{j}, j=1, \ldots, n$, are now the complex coordinates on $V$ with respect to this basis.

Now, we quantize the moment map $\mu$ according to (2.7) and denote the quantized moment map by $\widehat{\mu}$ as follows:

$$
\begin{aligned}
\widehat{\mu}: & =\left[\begin{array}{c}
\widehat{x}_{1} \\
\vdots \\
\widehat{y}_{n}
\end{array}\right]\left(\widehat{x}_{1}, \ldots, \widehat{y}_{n}\right) J_{n}=\left[\begin{array}{c}
x \\
-\mathrm{i} \partial_{x}
\end{array}\right]\left({ }^{t} x,-\mathrm{i}{ }^{t} \partial_{x}\right) J_{n} \\
& =\left[\begin{array}{cc}
\mathrm{i} x^{t} \partial_{x} & x^{t} x \\
\partial_{x}{ }^{t} \partial_{x} & -\mathrm{i} \partial_{x}{ }^{t} x
\end{array}\right]
\end{aligned}
$$

with $x={ }^{t}\left(x_{1}, \ldots, x_{n}\right)$ and $\partial_{x}={ }^{t}\left(\partial_{x_{1}}, \ldots, \partial_{x_{n}}\right)$.

Let $\mathcal{P}(V)$ denote the space of complex coefficient polynomial functions on $V$, i.e., $\mathcal{P}(V)=\mathbb{C}\left[x_{1}, \ldots, x_{n}\right]$, and $\mathcal{P} \mathcal{D}(V)$ the ring of polynomial coefficient differential operators on $V$. Thus each entry of $\widehat{\mu}$ is an element of $\mathcal{P D}(V)$.

Theorem 2.3. For $X \in \mathfrak{g}=\mathfrak{s p}_{n}$, set $\pi(X)=\mathrm{i}\langle\widehat{\mu}, X\rangle$. Then the map

$$
\pi: \mathfrak{g} \rightarrow \mathcal{P D}(V)
$$

is a Lie algebra homomorphism. In terms of the basis (2.1), it is given by

$$
\pi(X)=\left\{\begin{array}{lll}
-\frac{1}{2}\left(x_{i} \partial_{x_{j}}+\partial_{x_{j}} x_{i}\right) & \text { if } & X=X_{i, j}^{0} ; \\
\mathrm{i} \partial_{x_{i}} \partial_{x_{j}} & \text { if } & X=X_{i, j}^{+} \\
\mathrm{i} x_{i} x_{j} & \text { if } & X=X_{i, j}^{-}
\end{array}\right.
$$

Proof. Of course, one can verify the commutation relations among the explicit form (2.10), which can be easily deduced from (2.9), coincides with those of the basis $\left\{X_{i, j}^{\star}\right\}$ for $\mathrm{g}$. However, we will give another proof in the following.

The moment map $\mu$ induces a Lie algebra homomorphism from $\mathfrak{g}_{0}$ to $C^{\infty}(W)$, i.e., if we write $H_{X}:=\langle\mu, X\rangle$ for $X \in \mathfrak{g}_{0}$, then we have

$$
\left\{H_{X}, H_{Y}\right\}=H_{[X, Y]} \quad\left(X, Y \in \mathfrak{g}_{0}\right) .
$$

Taking account of the facts that both Poisson bracket and commutator are derivations, one sees that the relation (2.11) implies that

$$
\left[\widehat{H}_{X}, \widehat{H}_{Y}\right]=-\mathrm{i} \widehat{H}_{[X, Y]}
$$

as required in (1.2), since each function $H_{X}$ is quadratic in the coordinate functions $x_{i}, y_{j}$ for any $X \in \mathfrak{g}_{0}$ (see [CG97]) and the commutators among $\widehat{x}_{i}$ and $\widehat{y}_{j}$ are in the center of $\mathcal{P D}(V)$ for $i, j=1, \ldots, n$. Hence, it follows from $\pi(X)=\mathrm{i} \widehat{H}_{X}$ that

$$
[\pi(X), \pi(Y)]=\pi([X, Y]) \quad\left(X, Y \in \mathfrak{g}_{0}\right) .
$$


Now, extend the result to the complexification by linearity.

Remark 2.4. By (2.9), one can rewrite $\pi(X)=\mathrm{i}\langle\widehat{\mu}, X\rangle, X \in \mathfrak{g}$, as follows:

$$
\begin{aligned}
\pi(X) & =\frac{\mathrm{i}}{2} \operatorname{tr}(\widehat{\mu} X)=\frac{\mathrm{i}}{2} \operatorname{tr}\left(\left[\begin{array}{c}
x \\
-\mathrm{i} \partial_{x}
\end{array}\right]\left({ }^{t} x,-\mathrm{i}{ }^{t} \partial_{x}\right) J_{n} X\right) \\
& =\frac{\mathrm{i}}{2}\left(\mathrm{i}^{t} \partial_{x},{ }^{t} x\right) X\left[\begin{array}{c}
x \\
-\mathrm{i} \partial_{x}
\end{array}\right],
\end{aligned}
$$

where the last equality follows from the fact that $X$ is a member of $\mathfrak{g}$. Namely, our quantized moment map $\widehat{\mu}$ is essentially identical to the map $\varphi$ given in Example of [KV95, Chap. I, §6]. This observation was the original motivation of the present work.

It is well known that the irreducible decomposition of the representation $(\pi, \mathcal{P}(V))$ of $\mathfrak{g}$ is given by $\mathcal{P}(V)=\mathcal{P}(V)_{+} \oplus \mathcal{P}(V)_{-}$, where $\mathcal{P}(V)_{+}$and $\mathcal{P}(V)_{-}$are the subspaces consisting of even polynomials $f(x)$ satisfying $f(-x)=f(x)$ and of odd polynomials $f(x)$ satisfying $f(-x)=-f(x)$ respectively. It is also well known that this phenomena can be explained by the type of representations of $\mathrm{O}(1)$ which acts on $V$ on the right.

2.2. Let us consider the vector space $W^{k}:=W \oplus \cdots \oplus W$, the direct sum of $k$ copies of $W=\mathbb{R}^{2 n}$, which can be identified with $\operatorname{Mat}_{2 n \times k}(\mathbb{R})$. It is a symplectic vector space equipped with symplectic form $\omega_{k}$ given by

$$
\omega_{k}(v, w)=\operatorname{tr}\left({ }^{t} v J_{n} w\right) \quad\left(v, w \in W^{k}\right) .
$$

Let $e_{i, a}$ denote the matrix unit of size $2 n \times k$ for $i=1, \ldots, n$ and $a=1, \ldots, k$. Under the identification $e_{i, a} \leftrightarrow \partial_{x_{i, a}}$ and $e_{\bar{l}, a} \leftrightarrow \partial_{y_{i, a}}$, we write $v={ }^{t}\left[x_{1}, \ldots, x_{n}, y_{1}, \ldots, y_{n}\right] \in W^{k}$ with $x_{i}=\left(x_{i, 1}, \ldots, x_{i, k}\right)$ and $y_{i}=\left(y_{i, 1}, \ldots, y_{i, k}\right)$ being row vectors 1 of size $k$ for $i=1, \ldots, n$. Then $\omega_{k}$ is given by

$$
\omega_{k}=\sum_{1 \leqslant i \leqslant n, 1 \leqslant a \leqslant k} \mathrm{~d} x_{i, a} \wedge \mathrm{d} y_{i, a}
$$

at $v={ }^{t}\left[x_{1}, \ldots, y_{n}\right]$. Note that $G=\operatorname{Sp}(n, \mathbb{R})$ acts on $W^{k}=\operatorname{Mat}_{2 n \times k}(\mathbb{R})$ on the left, while the real orthogonal group $\mathrm{O}(k)$ acts on the right. Both actions are symplectic.

For brevity, let us write $x_{\star} \cdot y_{\star}=\sum_{a=1}^{k} x_{\star, a} y_{\star, a}$, the standard inner product between two row vectors $x_{\star}=\left(x_{\star, 1}, \ldots, x_{\star, k}\right)$ and $y_{\star}=\left(y_{\star, 1}, \ldots, y_{\star, k}\right)$ of size $k$ in what follows.

Proposition 2.5. Let $\left(W^{k}, \omega_{k}\right)$ be the symplectic G-vector space. Then the moment map $\mu: W^{k} \rightarrow \mathfrak{g}_{0}^{*} \simeq \mathfrak{g}_{0}$ is given by

$$
\mu(v)=v^{t} v J_{n}
$$

\footnotetext{
${ }^{1}$ More precisely, one should write an element $v \in W^{k}=\operatorname{Mat}_{2 n \times k}(\mathbb{R})$ as $v={ }^{t}\left[{ }^{t} x_{1}, \ldots,{ }^{t} x_{n},{ }^{t} y_{1}, \ldots,{ }^{t} y_{n}\right]$; however, we will adopt this abbreviated notation in what follows.
} 


$$
=\left[\begin{array}{cccc|cccc}
-x_{1} \cdot y_{1} & -x_{1} \cdot y_{2} & \cdots & -x_{1} \cdot y_{n} & x_{1} \cdot x_{1} & x_{1} \cdot x_{2} & \cdots & x_{1} \cdot x_{n} \\
-x_{2} \cdot y_{1} & -x_{2} \cdot y_{2} & \cdots & -x_{2} \cdot y_{n} & x_{2} \cdot x_{1} & x_{2} \cdot x_{2} & \cdots & x_{2} \cdot x_{n} \\
\vdots & \vdots & \ddots & \vdots & \vdots & \vdots & \ddots & \vdots \\
-x_{n} \cdot y_{1} & -x_{n} \cdot y_{2} & \cdots & -x_{n} \cdot y_{n} & x_{n} \cdot x_{1} & x_{n} \cdot x_{2} & \cdots & x_{n} \cdot x_{n} \\
\hline-y_{1} \cdot y_{1} & -y_{1} \cdot y_{2} & \cdots & -y_{1} \cdot y_{n} & y_{1} \cdot x_{1} & y_{1} \cdot x_{2} & \cdots & y_{1} \cdot x_{n} \\
-y_{2} \cdot y_{1} & -y_{2} \cdot y_{2} & \cdots & -y_{2} \cdot y_{n} & y_{2} \cdot x_{1} & y_{2} \cdot x_{2} & \cdots & y_{2} \cdot x_{n} \\
\vdots & \vdots & \ddots & \vdots & \vdots & \vdots & \ddots & \vdots \\
-y_{n} \cdot y_{1} & -y_{n} \cdot y_{2} & \cdots & -y_{n} \cdot y_{n} & y_{n} \cdot x_{1} & y_{n} \cdot x_{2} & \cdots & y_{n} \cdot x_{n}
\end{array}\right]
$$

for $v={ }^{t}\left[x_{1}, \ldots, x_{n}, y_{1}, \ldots, y_{n}\right] \in W^{k}$. In particular, $\mu$ is $G$-equivariant and is $\mathrm{O}(k)$ invariant.

Proof. In the agreements that $x_{i}, y_{i}, \partial_{x_{i}}$ and $\partial_{y_{i}}$ denote row vectors and that the products stand for the inner product of row vectors, a simple calculation shows that the vector fields on $W^{k}$ generated by the basis $(2.1)$ for $\mathfrak{g}_{0}=\mathfrak{s p}(n, \mathbb{R})$ are given by the same formulae as in Lemma 2.1, and thus the same argument given in the proof of Proposition 2.2 produces the result.

It follows from (2.12) that the Poisson brackets among the coordinate functions $x_{i, a}, y_{i, a}, i=1, \ldots, n ; a=1, \ldots, k$, are given by

$$
\left\{x_{i, a}, y_{j, b}\right\}=\delta_{i, j} \delta_{a, b}
$$

and all other brackets vanish. Therefore, we quantize them by assigning

$$
\widehat{x}_{i, a}=x_{i, a} \text { and } \widehat{y}_{i, a}=-\mathrm{i} \partial_{x_{i, a}}
$$

for $i=1, \ldots, n$ and $a=1, \ldots, k$.

Let $V^{k}$ denote the direct sum $V \oplus \cdots \oplus V$ (k copies) with $V$ given in (2.8). Since $V^{k}$ can be identified with $\operatorname{Mat}_{n \times k}(\mathbb{C})$, the upper half of $W_{\mathbb{C}}^{k}=\operatorname{Mat}_{2 n \times k}(\mathbb{C})$, we write an element of $V^{k}$ as $x=\left(x_{i, a}\right)_{i=1, \ldots, n ; a=1, \ldots, k}$. Let $\mathcal{P}\left(V^{k}\right)=\mathbb{C}\left[x_{i, a} ; i=1, \ldots, n, a=1, \ldots, k\right]$ be the algebra of complex polynomial functions on $V^{k}$, and $\mathcal{P} \mathcal{D}\left(V^{k}\right)$ the ring of polynomial coefficient differential operators on $V^{k}$. Note that $x_{i, a}$ 's are now complex variables and that the complex general linear group $\mathrm{GL}_{k}$ acts on $V^{k}$ by matrix multiplication on the right, and thus on $\mathcal{P}\left(V^{k}\right)$ by right translation:

$$
\rho(g) f(x):=f(x g) \quad\left(g \in \mathrm{GL}_{k}, f \in \mathcal{P}\left(V^{k}\right)\right) .
$$

The right-action of $\mathrm{GL}_{k}$ on $V^{k}$ is the restriction of the one on $W_{\mathbb{C}}^{k}$. (2.9):

The quantized moment map $\widehat{\mu}$ in this case is also given by the same formula as

$$
\widehat{\mu}=\left[\begin{array}{cc}
\mathrm{i} x^{t} \partial_{x} & x^{t} x \\
\partial_{x}{ }^{t} \partial_{x} & -\mathrm{i} \partial_{x}^{t} x
\end{array}\right]
$$

In this case, however, $x$ and $\partial_{x}$ are $n \times k$-matrices whose $(i, a)$-th entries are the multiplication operator $x_{i, a}$ and the differential operator $\partial_{x_{i, a}}$ for $i=1, \ldots, n$ and $a=1, \ldots, k$, respectively. 
Lemma 2.6. For $x=\left(x_{i, a}\right)_{i=1, \ldots, n ; a=1, \ldots, k} \in V^{k}$ and $g \in \mathrm{GL}_{k}$, the following relations hold in $\operatorname{End}\left(\mathcal{P}\left(V^{k}\right)\right)$ :

$$
\begin{aligned}
& \rho(g)^{-1} \partial_{x_{i, a}} \rho(g)=\sum_{b} g_{a b} \partial_{x_{i, b}}, \\
& \rho(g)^{-1} x_{i, a} \rho(g)=\sum_{b} g^{b a} x_{i, b},
\end{aligned}
$$

where $g=\left(g_{a b}\right)$ and $g^{-1}=\left(g^{a b}\right)$.

Proof. Since $\partial_{x_{i, a}}$ is identified with $e_{i, a} \in \operatorname{Mat}_{n \times k}(\mathbb{C})$, one sees that

$$
\left(\partial_{i, a}(\rho(g) f)\right)(x)=\left.\frac{\mathrm{d}}{\mathrm{d} t}\right|_{t=0} f\left(x g+t e_{i, a} g\right)=\sum_{b=0}^{k} g_{a b} \frac{\partial f}{\partial x_{i, b}}(x g),
$$

and hence

$$
\left(\rho(g)^{-1} \partial_{x_{i, a}} \rho(g)\right) f=\sum_{b=1}^{k} g_{a b} \frac{\partial f}{\partial x_{i, b}}
$$

for $f \in \mathcal{P}\left(V^{k}\right)$. Thus one obtains (2.15).

On the other hand, since

$$
\left(\rho(g)^{-1}\left(x_{i, a} f\right)\right)(x)=\left(\sum_{b=1}^{k} x_{i, b} g^{b a}\right) f\left(x g^{-1}\right)
$$

one has

$$
\left(\rho(g)^{-1} x_{i, a} \rho(g)\right) f=\left(\sum_{b=1}^{k} g^{b a} x_{i, b}\right) f
$$

and (2.16).

Let us abbreviate as $\rho(g) a \rho(g)^{-1}=: \operatorname{Ad}_{\rho(g)} a$ for $a \in \mathcal{P D}\left(V^{k}\right)$ and $g \in \mathrm{GL}_{k}$. Moreover, for a given matrix $A=\left(a_{i j}\right)$ with $a_{i j} \in \mathcal{P D}\left(V^{k}\right)$, let us denote by $\operatorname{Ad}_{\rho(g)} A=$ $\left(\operatorname{Ad}_{\rho(g)} a_{i j}\right)$, the matrix whose $(i, j)$-th entries are equal to $\operatorname{Ad}_{\rho(g)} a_{i j}$.

Corollary 2.7. For $X \in \mathfrak{g}=\mathfrak{s p}_{n}$, set $\pi(X)=\mathrm{i}\langle\widehat{\mu}, X\rangle$. Then the map

$$
\pi: \mathfrak{g} \rightarrow \mathcal{P} \mathcal{D}\left(V^{k}\right)
$$

is a Lie algebra homomorphism. In terms of the basis (2.1), it is given by

$$
\pi(X)=\left\{\begin{array}{lll}
-\frac{1}{2} \sum_{a=1}^{k}\left(x_{i, a} \partial_{x_{j, a}}+\partial_{x_{j, a}} x_{i, a}\right) & \text { if } & X=X_{i, j}^{0} \\
\mathrm{i} \sum_{a=1}^{k} \partial_{x_{i, a}} \partial_{x_{j, a}} & \text { if } & X=X_{i, j}^{+} \\
\mathrm{i} \sum_{a=1}^{k} x_{i, a} x_{j, a} & \text { if } & X=X_{i, j}^{-}
\end{array}\right.
$$

Moreover, $\pi(X)$ commutes with the action of the complex orthogonal group $2 \mathrm{O}_{k}$, i.e., $\pi(X) \in \mathcal{P D}\left(V^{k}\right)^{\mathrm{O}_{k}}$ for all $X \in \mathfrak{s p}_{n}$.

\footnotetext{
${ }^{2}$ We realize the complex orthogonal group as $\mathrm{O}_{k}=\left\{g \in \mathrm{GL}_{k} ;{ }^{t} g g=1_{k}\right\}$ in this section.
} 
Proof. The same argument as in the proof of Theorem 2.3 shows that $\pi: \mathfrak{g} \rightarrow \mathcal{P D}\left(V^{k}\right)$ is a Lie algebra homomorphism and that (2.17) holds.

For the last statement, it follows from Lemma 2.6 that

$$
\operatorname{Ad}_{\rho(g)^{-1}} x_{i}=x_{i} g^{-1} \quad \text { and } \quad \operatorname{Ad}_{\rho(g)^{-1}} \partial_{x_{i}}=\partial_{x_{i}}{ }^{t} g
$$

with $x_{i}=\left(x_{i, 1}, \ldots, x_{i, k}\right)$ and $\partial_{x_{i}}=\left(\partial_{x_{i, 1}}, \ldots, \partial_{x_{i, k}}\right)$ for $g \in \mathrm{GL}_{k}$. Hence, if $g \in \mathrm{O}_{k}$ then one has

$$
\left[\begin{array}{c}
\operatorname{Ad}_{\rho(g)^{-1}} x \\
-\mathbf{i} \mathbf{A d}_{\rho(g)^{-1}} \partial
\end{array}\right]=\left[\begin{array}{c}
x \\
-\mathrm{i} \partial
\end{array}\right]{ }^{t} g
$$

with $x={ }^{t}\left[x_{1}, \ldots, x_{n}\right]$ and $\partial={ }^{t}\left[\partial_{x_{1}}, \ldots, \partial_{x_{n}}\right]$, since ${ }^{t} g=g^{-1}$. Therefore,

$$
\begin{aligned}
& \operatorname{Ad}_{\rho(g)^{-1}} \widehat{\mu}=\left[\begin{array}{cc}
\mathrm{i} \operatorname{Ad}_{\rho(g)^{-1}}\left(x^{t} \partial_{x}\right) & \operatorname{Ad}_{\rho(g)^{-1}}\left(x^{t} x\right) \\
\operatorname{Ad}_{\rho(g)^{-1}}\left(\partial_{x}{ }^{t} \partial_{x}\right) & -\mathrm{i} \operatorname{Ad}_{\rho(g)^{-1}}\left(\partial_{x}{ }^{t} x\right)
\end{array}\right] \\
& =\left[\begin{array}{cc}
\mathrm{i} \mathrm{Ad}_{\rho(g)^{-1}} x^{t}\left(\mathbf{A d}_{\rho(g)^{-1}} \partial_{x}\right) & \mathbf{A d}_{\rho(g)^{-1}} x^{t}\left(\mathbf{A d}_{\rho(g)^{-1}} x\right) \\
\left.\operatorname{Ad}_{\rho(g)^{-1}} \partial_{x}{ }^{t} \mathbf{A d}_{\rho(g)^{-1}} \partial_{x}\right) & -\mathrm{i} \mathbf{A d}_{\rho(g)^{-1}} \partial_{x}{ }^{t}\left(\mathbf{A d}_{\rho(g)^{-1}} x\right)
\end{array}\right] \\
& =\left[\begin{array}{c}
\mathbf{A d}_{\rho(g)^{-1}} x \\
-\mathbf{i} \mathbf{A d}_{\rho(g)^{-1}} \partial_{x}
\end{array}\right]\left[{ }^{t}\left(\mathbf{A d}_{\rho(g)^{-1}} x\right), \quad-\mathrm{i}^{t}\left(\mathbf{A d}_{\rho(g)^{-1}} \partial_{x}\right)\right] J_{n} \\
& =\left[\begin{array}{c}
x \\
-\mathrm{i} \partial
\end{array}\right]{ }^{t} g g\left[{ }^{t} x,-\mathrm{i}^{t} \partial\right] J_{n}=\widehat{\mu} \text {. }
\end{aligned}
$$

This completes the proof.

It is well known that the irreducible decomposition of $\mathcal{P}\left(V^{k}\right)$ under the joint action of $\left(\mathfrak{s p}_{n}, \mathrm{O}_{k}\right)$ is given by

$$
\mathcal{P}\left(V^{k}\right) \simeq \sum_{\sigma \in \widehat{\mathrm{O}}_{k}, L(\sigma) \neq\{0\}} L(\sigma) \otimes V_{\sigma},
$$

where $V_{\sigma}$ is a representative of the class $\sigma \in \widehat{\mathrm{O}}_{k}$, the set of all equivalence classes of the finite-dimensional irreducible representation of $\mathrm{O}_{k}$, and $L(\sigma):=\operatorname{Hom}_{\mathrm{O}_{k}}\left(V_{\sigma}, \mathcal{P}\left(V^{k}\right)\right)$ which is an infinite-dimensional irreducible representation of $\mathfrak{s p}_{n}$. Moreover, it is also known that the action $\pi$ restricted to $¥$ lifts to the double cover $\tilde{K}_{\mathbb{C}}$ of the complexification $K_{\mathbb{C}}$ of the maximal compact subgroup $K$ of $G=\operatorname{Sp}(n, \mathbb{R})$, which implies that $L(\sigma)$ is an irreducible $\left(\mathfrak{g}, \tilde{K}_{\mathbb{C}}\right)$-module.

Note that our realization of the representation $\pi$ is the Schrödinger model of the oscillator representation of $\mathfrak{g}=\mathfrak{s p}_{n}$; we will need another realization of the representation in $\S 5$, i.e., the Fock model.

\section{REDUCTIVE DUAL PAIR $\left(\mathfrak{u}(p, q), \mathrm{GL}_{k}\right)$}

Let $G$ denote the indefinite unitary group defined by

$$
\mathrm{U}(p, q)=\left\{g \in \mathrm{GL}_{n}(\mathbb{C}) ; g^{*} I_{p, q} g=I_{p, q}\right\}
$$


with $I_{p, q}=\left[\begin{array}{ll}1_{p} & \\ & -1_{q}\end{array}\right]$, and put $n=p+q$ only in this section for brevity. Set $\mathfrak{g}_{0}=\mathfrak{u}(p, q)$, the Lie algebra of $G$, and take a basis for $\mathfrak{g}_{0}$ as follows:

$$
\begin{aligned}
X_{i, j}^{c}=E_{i, j}-E_{j, i} & (1 \leqslant i<j \leqslant p, \text { or } p+1 \leqslant i<j \leqslant n), \\
Y_{i, j}^{c}=\mathrm{i}\left(E_{i, j}+E_{j, i}\right) & (1 \leqslant i \leqslant j \leqslant p, \text { or } p+1 \leqslant i \leqslant j \leqslant n), \\
X_{i, j}^{n}=E_{i, \bar{J}}+E_{\bar{\jmath}, i} & (1 \leqslant i \leqslant p, 1 \leqslant j \leqslant q), \\
Y_{i, j}^{n}=\mathrm{i}\left(E_{i, \bar{j}}-E_{\bar{\jmath}, i}\right) & (1 \leqslant i \leqslant p, 1 \leqslant j \leqslant q),
\end{aligned}
$$

where $E_{i, j}$ denotes the matrix unit of size $n \times n$. Note that $E_{i, j}, i, j=1, \ldots, n$, form a basis for $\mathfrak{g}=\mathfrak{g l}_{n}$, the complexified Lie algebra of $\mathfrak{g}_{0}=\mathfrak{u}(p, q)$.

3.1. Let $W=\left(\mathbb{C}^{n}\right)_{\mathbb{R}}$, the underlying real vector space of the complex vector space $\mathbb{C}^{n}$, and $H: \mathbb{C}^{n} \times \mathbb{C}^{n} \rightarrow \mathbb{C}$ the indefinite Hermitian form given by

$$
H(z, w):=z^{*} I_{p, q} w \quad\left(z, w \in \mathbb{C}^{n}\right) .
$$

We regard $W$ as symplectic manifold with symplectic form $\omega=\operatorname{Im} H$, where $\operatorname{Im} H$ stands for the imaginary part of $H$. Under the identification $e_{j} \leftrightarrow \partial_{x_{j}}$ and i $e_{j} \leftrightarrow \partial_{y_{j}}$ for $j=1, \ldots, n$, it is explicitly given by

$$
\omega=\sum_{j=1}^{n} \epsilon_{j} \mathrm{~d} x_{j} \wedge \mathrm{d} y_{j}
$$

at $z=x+\mathrm{i} y \in W$ with $x={ }^{t}\left(x_{1}, \ldots, x_{n}\right), y={ }^{t}\left(y_{1}, \ldots, y_{n}\right) \in \mathbb{R}^{n}$, where

$$
\epsilon_{j}:=\left\{\begin{aligned}
1 & (j=1, \ldots, p) \\
-1 & (j=p+1, \ldots, n) .
\end{aligned}\right.
$$

Then $(W, \omega)$ is a symplectic $G$-manifold since the natural action of $G$ on $\mathbb{C}^{n}$ preserves the Hermitian form $H$.

Lemma 3.1. The vector fields on $W$ generated by the basis (3.1) for $\mathfrak{g}_{0}=\mathfrak{u}(p, q)$ in the sense of (1.4) are given by

$$
\begin{aligned}
& \left(X_{i, j}^{c}\right)_{W}=-x_{j} \partial_{x_{i}}-y_{j} \partial_{y_{i}}+x_{i} \partial_{x_{j}}+y_{i} \partial_{y_{j}}, \\
& \left(Y_{i, j}^{c}\right)_{W}=y_{j} \partial_{x_{i}}-x_{j} \partial_{y_{i}}+y_{i} \partial_{x_{j}}-x_{i} \partial_{y_{j}}, \\
& \left(X_{i, j}^{n}\right)_{W}=-x_{\bar{J}} \partial_{x_{i}}-x_{i} \partial_{x_{j}}-y_{\bar{J}} \partial_{y_{i}}-y_{i} \partial_{y_{j}}, \\
& \left(Y_{i, j}^{n}\right)_{W}=y_{j} \partial_{x_{i}}-x_{\bar{J}} \partial_{y_{i}}-y_{i} \partial_{x_{j}}+x_{i} \partial_{y_{j}} .
\end{aligned}
$$

Note that the unitary group $\mathrm{U}(1)$ also acts on $W$ symplectically on the right.

Proposition 3.2. Let $(W, \omega)$ be as above and $G=\mathrm{U}(p, q)$. Then the moment map $\mu: W \rightarrow \mathfrak{g}_{0}^{*} \simeq \mathfrak{g}_{0}$ is given by

$$
\mu(z)=-\frac{\mathrm{i}}{2} z z^{*} I_{p, q}
$$

for $z=x+\mathrm{i} y \in W$ with $x={ }^{t}\left(x_{1}, \ldots, x_{n}\right), y={ }^{t}\left(y_{1}, \ldots, y_{n}\right) \in \mathbb{R}^{n}$. In particular, $\mu$ is $G$-equivariant and is $\mathrm{U}(1)$-invariant. 
Proof. It follows from Lemma 3.1 that

$$
\langle\mu, X\rangle=\left\{\begin{array}{llc}
\epsilon_{i}\left(x_{i} y_{j}-x_{j} y_{i}\right) & \text { if } & X=X_{i, j}^{c} \\
\epsilon_{i}\left(x_{i} x_{j}+y_{i} y_{j}\right) & \text { if } & X=Y_{i, j}^{c} \\
x_{i} y_{\bar{j}}-x_{\bar{J}} y_{i} & \text { if } & X=X_{i, j}^{n} \\
x_{i} x_{\bar{J}}+y_{i} y_{\bar{J}} & \text { if } & X=Y_{i, j}^{n}
\end{array}\right.
$$

which can be rewritten in terms of the complex coordinates defined by $z_{j}=x_{j}+\mathrm{i} y_{j}$ $(j=1, \ldots, n)$ and their complex conjugates as

$$
\langle\mu, X\rangle=\left\{\begin{array}{lll}
\frac{\mathrm{i}}{2} \epsilon_{i}\left(z_{i} \bar{z}_{j}-z_{j} \bar{z}_{i}\right) & \text { if } & X=X_{i, j}^{c} ; \\
\frac{1}{2} \epsilon_{i}\left(z_{i} \bar{z}_{j}+z_{j} \bar{z}_{i}\right) & \text { if } & X=Y_{i, j}^{c} ; \\
\frac{\mathrm{i}}{2}\left(z_{i} \bar{z}_{\bar{j}}-z_{j} \bar{z}_{i}\right) & \text { if } & X=X_{i, j}^{n} \\
\frac{1}{2}\left(z_{i} \bar{z}_{\bar{j}}+z_{\bar{j}} \bar{z}_{i}\right) & \text { if } & X=Y_{i, j}^{n} .
\end{array}\right.
$$

Hence,

$$
\begin{aligned}
\mu(z) & =\sum_{i<j}\left\langle\mu, X_{i, j}^{c}\right\rangle\left(X_{i, j}^{c}\right)^{\vee}+\sum_{i \leqslant j}\left\langle\mu, Y_{i, j}^{c}\right\rangle\left(Y_{i, j}^{c}\right)^{\vee}+\sum_{i, j}\left\langle\mu, X_{i, j}^{n}\right\rangle\left(X_{i, j}^{n}\right)^{\vee}+\sum_{i, j}\left\langle\mu, Y_{i, j}^{n}\right\rangle\left(Y_{i, j}^{n}\right)^{\vee} \\
& =-\frac{\mathrm{i}}{2} \sum_{1 \leqslant i, j \leqslant p} z_{i} \bar{z}_{j} E_{i, j}+\frac{\mathrm{i}}{2} \sum_{1 \leqslant i, j \leqslant q} z_{\bar{l}} \bar{z}_{\bar{j}} E_{\bar{l}, \bar{j}}+\frac{\mathrm{i}}{2} \sum_{1 \leqslant i \leqslant p, 1 \leqslant j \leqslant q} z_{i} \bar{z}_{\bar{j}} E_{i, \bar{j}}-\frac{\mathrm{i}}{2} \sum_{1 \leqslant i \leqslant q, 1 \leqslant j \leqslant p} z_{\bar{l}} \bar{z}_{j} E_{\bar{l}, j} \\
& =-\frac{\mathrm{i}}{2} z z^{*} I_{p, q},
\end{aligned}
$$

with $z={ }^{t}\left(z_{1}, \ldots, z_{n}\right)$.

The U(1)-invariance of $\mu$ is obvious, and the $G$-equivariance can be verified as follows:

$$
\mu(g z)=-\frac{\mathrm{i}}{2}(g z)(g z)^{*} I_{p, q}=-\frac{\mathrm{i}}{2} g z z^{*} g^{*} I_{p, q}=\operatorname{Ad}(g) \mu(z)
$$

since $g^{*} I_{p, q}=I_{p, q} g^{-1}$ for $g \in \mathrm{U}(p, q)$.

It follows from (3.2) that the Poisson brackets among the real coordinate functions $x_{i}, y_{i}, i=1, \ldots, n$, are given by

$$
\left\{x_{i}, y_{j}\right\}=-\epsilon_{i} \delta_{i, j} \quad(i, j=1,2, \ldots, n),
$$

and all other brackets vanish. In terms of the complex coordinates $z_{j}=x_{j}+\mathrm{i} y_{j}, j=$ $1,2, \ldots, n$, and their conjugates, it follows from (3.8) that the Poisson brackets among $z_{j}$ and $\bar{z}_{j}$ are given by

$$
\left\{z_{i}, \bar{z}_{j}\right\}=2 \mathrm{i} \epsilon_{i} \delta_{i, j}, \quad\left\{z_{i}, z_{j}\right\}=\left\{\bar{z}_{i}, \bar{z}_{j}\right\}=0
$$

for $i, j=1,2, \ldots, n$. In view of (3.9) we quantize $z_{i}$ and $\bar{z}_{i}$ by assigning

$$
\widehat{z}_{i}=z_{i}, \quad \widehat{\bar{z}}_{i}=-2 \epsilon_{i} \partial_{z_{i}},
$$

so that they satisfy

$$
\left[\widehat{z}_{i}, \widehat{\bar{z}}_{j}\right]=2 \epsilon_{i} \delta_{i, j}, \quad\left[\widehat{z}_{i}, \widehat{z}_{j}\right]=\left[\widehat{\bar{z}}_{i}, \widehat{\bar{z}}_{j}\right]=0
$$


for $i, j=1,2, \ldots, n$. Therefore, we quantize the moment map $\mu$ and denote the quantized moment map by $\widehat{\mu}$ as follows:

$$
\widehat{\mu}=-\frac{\mathrm{i}}{2}\left[\begin{array}{c}
\widehat{z}_{1} \\
\vdots \\
\widehat{\bar{z}}_{n}
\end{array}\right]\left(\widehat{\bar{z}}_{1}, \ldots, \widehat{\bar{z}}_{n}\right) I_{p, q}=\mathrm{i}\left[\begin{array}{c}
z_{1} \\
\vdots \\
z_{n}
\end{array}\right]\left(\partial_{z_{1}}, \ldots, \partial_{z_{n}}\right)=\mathrm{i} z^{t} \partial_{z}
$$

with $z={ }^{t}\left(z_{1}, \ldots, z_{n}\right)$ and $\partial_{z}={ }^{t}\left(\partial_{z_{1}}, \ldots, \partial_{z_{n}}\right)$. Note that the quantization (3.10) corresponds to taking a complex Lagrangian subspace $V^{\prime}$ given by

$$
V^{\prime}:=\left\langle\frac{1}{2}\left(e_{1}-\mathrm{i} I e_{1}\right), \ldots, \frac{1}{2}\left(e_{n}-\mathrm{i} I e_{n}\right)\right\rangle_{\mathbb{C}} \subset W_{\mathbb{C}},
$$

where $I$ denotes the complex structure on $W$ defined by $e_{j} \mapsto \mathrm{i} e_{j}$, i $e_{j} \mapsto-e_{j}$ for $j=1, \ldots, n$. The classical observables $z_{j}=x_{j}+\mathrm{i} y_{j}$ can be regarded as the coordinates on $V^{\prime}$ with respect to this basis under the identification $e_{j} \leftrightarrow \partial_{x_{j}}$ and i $e_{j} \leftrightarrow \partial_{y_{j}}, j=$ $1, \ldots, n$, and $V^{\prime}$ is naturally identified with $\mathbb{C}^{n}$. Let $\mathcal{P}\left(V^{\prime}\right)$ denote the algebra of complex coefficient polynomial functions on $V$, i.e., $\mathcal{P}\left(V^{\prime}\right)=\mathbb{C}\left[z_{1}, \ldots, z_{n}\right]$, and $\mathcal{P} \mathcal{D}\left(V^{\prime}\right)$ the ring of polynomial coefficient differential operators on $V^{\prime}$.

Theorem 3.3. For $X \in \mathfrak{g}=\mathfrak{g l}_{n}$, set $\pi(X)=\mathrm{i}\langle\widehat{\mu}, X\rangle$. Then the map

$$
\pi: \mathfrak{g} \rightarrow \mathcal{P D}\left(V^{\prime}\right)
$$

is a Lie algebra homomorphism. In terms of the basis $\left\{E_{i, j}\right\}$ for $\mathfrak{g}$, it is given by

$$
\pi\left(E_{i, j}\right)=-z_{j} \partial_{z_{i}}
$$

for $i, j=1, \ldots, n$.

Proof. The same argument as in Theorem 2.3 shows that $\pi$ is a Lie algebra homomorphism, and (3.14) follows immediately from (3.12).

It is clear from (3.14) that $\pi(X) \in \mathcal{P D}\left(V^{\prime}\right)^{\mathrm{GL}_{1}}$ for all $X \in \mathfrak{g}$, where $\mathrm{GL}_{1}$ acts on $V^{\prime}$ on the right.

3.2. Now let us consider $W^{k}$, the direct sum of $k$ copies of $W=\left(\mathbb{C}^{n}\right)_{\mathbb{R}}$, which is identified with the underlying real vector space of $\mathrm{Mat}_{n \times k}(\mathbb{C})$. It is equipped with a symplectic form $\omega_{k}$ given by

$$
\omega_{k}(z, w)=\operatorname{Im} \operatorname{tr}\left(z^{*} I_{p, q} w\right) \quad\left(z, w \in W^{k}\right),
$$

and is still acted on by $G=\mathrm{U}(p, q)$ symplectically by matrix multiplication on the left. Under the identification of $e_{i, a} \leftrightarrow \partial_{x_{i, a}}$ and i $e_{i, a} \leftrightarrow \partial_{y_{i, a}}$, we write an element of $W^{k}$ as $z={ }^{t}\left[z_{1}, \ldots, z_{n}\right]$, where $z_{i}=x_{i}+\mathrm{i} y_{i}$ are complex row vectors with $x_{i}=\left(x_{i, 1}, \ldots, x_{n, k}\right)$ and $y_{i}=\left(y_{i, 1}, \ldots, y_{i, k}\right)$ being real row vectors of size $k$ for $i=1, \ldots, n$. Then $\omega_{k}$ is given by

$$
\omega_{k}=\sum_{1 \leqslant i \leqslant n, 1 \leqslant a \leqslant k} \epsilon_{i} \mathrm{~d} x_{i, a} \wedge \mathrm{d} y_{i, a}
$$

at $z={ }^{t}\left[z_{1}, \ldots, z_{n}\right] \in W^{k}$. Note that $\mathrm{U}(p, q)$ acts on $W$ on the left, while $\mathrm{U}(k)$ acts on it on the right and that both actions are symplectic. 
Proposition 3.4. Let $\left(W^{k}, \omega_{k}\right)$ be the symplectic G-vector space as above. Then the moment map $\mu: W^{k} \rightarrow \mathfrak{g}_{0}^{*} \simeq \mathfrak{g}_{0}$ is given by the same formula as 3.5]

$$
\mu=-\frac{\mathrm{i}}{2} z z^{*} I_{p, q}
$$

with $z \in W^{k}=\operatorname{Mat}_{n \times k}(\mathbb{C})$. In particular, $\mu$ is $G$-equivariant and is $\mathrm{U}(k)$-invariant.

Proof. As in the proof of Proposition 2.5, if we regard $x_{i}, y_{i}, \partial_{x_{i}}$ and $\partial_{y_{i}}$ as row vectors and the products as the inner product on the space of row vectors, then similar argument to Proposition 3.2 shows that the moment map $\mu: W^{k} \rightarrow \mathfrak{g}_{0}$ is given by (3.5), with the understanding that $z \in \operatorname{Mat}_{n \times k}(\mathbb{C})$. The $\mathrm{U}(k)$-invariance is obvious, and the $G$ equivariance is verified as in Proposition 3.2.

It follows from (3.15) that the Poisson brackets among the real coordinate functions $x_{i, a}, y_{i, a}, i=1, \ldots, n ; a=1, \ldots, k$, are given by

$$
\left\{x_{i, a}, y_{j, b}\right\}=-\epsilon_{i} \delta_{i, j} \delta_{a, b} \quad(i, j=1, \ldots, n ; a, b=1, \ldots, k),
$$

and all other brackets vanish. It follows from (3.16) that the Poisson brackets among the complex coordinates $z_{j, a}=x_{j, a}+\mathrm{i} y_{j, a}$ and their conjugates are given by

$$
\left\{z_{i, a}, \bar{z}_{j, b}\right\}=2 \mathrm{i} \epsilon_{i} \delta_{i, j} \delta_{a, b}
$$

for $i, j=1, \ldots, n ; a, b=1, \ldots, k$, and all other brackets vanish. Therefore, we quantize $z_{i, a}$ and $\bar{z}_{i, a}$ by assigning

$$
\widehat{z}_{i, a}=z_{i, a}, \quad \widehat{\bar{z}}_{i, a}=-2 \epsilon_{i} \partial_{z_{i, a}},
$$

so that the nontrivial commutators are given by

$$
\left[\widehat{z}_{i, a}, \widehat{\bar{z}}_{j, b}\right]=2 \epsilon_{i} \delta_{i, j} \delta_{a, b} \text {. }
$$

Let $V^{\prime k}$ denote the direct sum of $k$ copies of $V^{\prime}$, with $V^{\prime}$ as in 3.13 . Since $V^{\prime k}$ can be identified with $\operatorname{Mat}_{n \times k}(\mathbb{C})$, we write an element of $V^{\prime k}$ as $z=\left(z_{i, a}\right)_{i=1, \ldots, n ; a=1, \ldots, k}$. Note then that $\mathrm{GL}_{k}$ acts on $V^{\prime k}$ by matrix multiplication on the right, and hence acts on $\mathcal{P}\left(V^{\prime k}\right)$ by right regular representation, which we denote also by $\rho$ as in (2.14). Let $\mathcal{P}\left(V^{\prime k}\right)=$ $\mathbb{C}\left[z_{i, a} ; i=1, \ldots, n, a=1, \ldots, k\right]$ be the algebra of complex polynomial functions on $V^{\prime k}$, and $\mathcal{P} \mathcal{D}\left(V^{\prime k}\right)$ the ring of polynomial coefficient differential operators on $V^{\prime k}$.

The quantized moment map $\widehat{\mu}$ is also given by the same formula as (3.12):

$$
\widehat{\mu}=\mathrm{i} z{ }^{t} \partial_{z} \text {. }
$$

In this case, however, $z$ and $\partial_{z}$ are $n \times k$-matrices whose $(i, a)$-th entries are the multiplication operator $z_{i, a}$ and the differential operator $\partial_{z_{i, a}}$ for $i=1, \ldots, n$ and $a=1, \ldots, k$, respectively.

Corollary 3.5. For $X \in \mathfrak{g}=\mathfrak{g l}_{n}$, set $\pi(X)=\mathrm{i}\langle\widehat{\mu}, X\rangle$. Then the map

$$
\pi: \mathfrak{g} \rightarrow \mathcal{P D}\left(V^{\prime k}\right)
$$

is a Lie algebra homomorphism. In terms of the basis $\left\{E_{i, j}\right\}$ for $\mathfrak{g}$, it is given by

$$
\pi\left(E_{i, j}\right)=-\sum_{a=1}^{k} z_{j, a} \partial_{z_{i, a}}
$$


for $i, j=1, \ldots, n$. Moreover, $\pi(X)$ commutes with the action of the complex general linear group $\mathrm{GL}_{k}$, i.e., $\pi(X) \in \mathcal{P} \mathcal{D}\left(V^{\prime k}\right)^{\mathrm{GL}_{k}}$ for all $X \in \mathfrak{g}$.

Proof. The first statement that $\pi$ is a Lie algebra homomorphism can be shown as in the proof of Theorem 2.3. It remains to show that $\widehat{\mu}$ commutes with the action of $\mathrm{GL}_{k}$, which can be done in the following way. By Lemma 2.6, one obtains that

$$
\operatorname{Ad}_{\rho(g)^{-1}} z=z g^{-1} \text { and } \operatorname{Ad}_{\rho(g)^{-1}} \partial_{z}=\partial_{z}{ }^{t} g
$$

from which it follows that

$$
\operatorname{Ad}_{\rho(g)^{-1}}\left(z^{t} \partial_{z}\right)=\left(\operatorname{Ad}_{\rho(g)^{-1}} z\right)^{t}\left(\mathbf{A d}_{\rho(g)^{-1}} \partial_{z}\right)=z^{-1} g^{t} \partial_{z}=z^{t} \partial_{z} .
$$

This completes the proof.

Similarly to the case of $\operatorname{Sp}(n, \mathbb{R})$, it is well known that the irreducible decomposition of $\mathcal{P}\left(V^{k}\right)$ under the joint action of $\left(\mathrm{gl}_{n}, \mathrm{GL}_{k}\right)$ is given by

$$
\mathcal{P}\left(V^{\prime k}\right) \simeq \sum_{\sigma \in \widehat{\mathrm{GL}}_{k}, L(\sigma) \neq\{0\}} L(\sigma) \otimes V_{\sigma},
$$

where $V_{\sigma}$ is a representative of the class $\sigma \in \widehat{\mathrm{GL}}_{k}$, the set of all equivalence classes of the finite-dimensional irreducible representation of $\mathrm{GL}_{k}$, and $L(\sigma):=\operatorname{Hom}_{\mathrm{GL}_{k}}\left(V_{\sigma}, \mathcal{P}\left(V^{\prime k}\right)\right)$ which is a finite-dimensional irreducible representation of $\mathfrak{g l}_{n}$. It is also well known that the action $\pi$ restricted to $\mathfrak{f}$ lifts to the complexification $K_{\mathbb{C}}$ of the maximal compact subgroup $K$ of $G=\mathrm{U}(p, q)$, which implies that $L(\sigma)$ is an irreducible $\left(\mathfrak{g}, K_{\mathbb{C}}\right)$-module.

\section{RedUCTIVE DUAL PAIR $\left(\mathfrak{o}^{*}(2 n), \mathrm{Sp}_{k}\right)$}

In this section, let $G$ denote the linear Lie group defined by

$$
\begin{aligned}
\mathrm{O}^{*}(2 n) & =\left\{g \in \mathrm{U}(n, n) ;{ }^{t} g S g=S\right\} \\
& =\left\{g \in \mathrm{GL}_{2 n}(\mathbb{C}) ; g^{*} I_{n, n} g=I_{n, n},{ }^{t} g S g=S\right\},
\end{aligned}
$$

where $S$ denotes the nondegenerate symmetric matrix $\left[\begin{array}{r}1_{n} \\ 1_{n}\end{array}\right]$ of size $2 n \times 2 n$. Set $\mathfrak{g}_{0}=$ $\mathfrak{D}^{*}(2 n)$, the Lie algebra of $G$, and take a basis for $\mathfrak{g}_{0}$ as follows:

$$
\begin{aligned}
X_{i, j}^{c}=E_{i, j}-E_{j, i}+E_{\bar{l}, \bar{j}}-E_{\bar{\jmath}, \bar{l}} & (1 \leqslant i<j \leqslant n), \\
Y_{i, j}^{c}=\mathrm{i}\left(E_{i, j}+E_{j, i}-E_{\bar{l}, \bar{j}}-E_{\bar{\jmath}, \bar{l}}\right) & (1 \leqslant i \leqslant j \leqslant n), \\
X_{i, j}^{n}=E_{i, \bar{j}}-E_{j, \bar{l}}-E_{\bar{l}, j}+E_{\bar{\jmath}, i} & (1 \leqslant i<j \leqslant n), \\
Y_{i, j}^{n}=\mathrm{i}\left(E_{i, \bar{j}}-E_{j, \bar{l}}+E_{\bar{l}, j}-E_{\bar{\jmath}, i}\right) & (1 \leqslant i<j \leqslant n),
\end{aligned}
$$

where $E_{i, j}$ denotes the matrix unit of size $2 n \times 2 n$. The complexified Lie algebra $\mathfrak{D}_{2 n}$ of $\mathfrak{g}_{0}=\mathfrak{o}^{*}(2 n)$ is realized as

$$
\mathfrak{D}_{2 n}=\left\{X \in \operatorname{Mat}_{2 n}(\mathbb{C}) ;{ }^{t} X S+S X=O\right\}
$$

in this section, which we will denote by $g$ below. It has a basis

$$
\begin{array}{ll}
X_{i, j}^{0}=E_{i, j}-E_{\bar{j}, \bar{l}} & (1 \leqslant i, j \leqslant n), \\
X_{i, j}^{+}=E_{i, \bar{j}}-E_{j, \bar{l}} & (1 \leqslant i<j \leqslant n), \\
X_{i, j}^{-}=E_{\bar{j}, i}-E_{\bar{l}, j} \quad(1 \leqslant i<j \leqslant n) .
\end{array}
$$


4.1. Let $W=\left(\mathbb{C}^{2 n}\right)_{\mathbb{R}}$ and $\omega=\operatorname{Im} H$, where $H: \mathbb{C}^{2 n} \times \mathbb{C}^{2 n} \rightarrow \mathbb{C}$ is the Hermitian form given by

$$
H(u, v)=u^{*} I_{n, n} v \quad\left(u, v \in \mathbb{C}^{2 n}\right) .
$$

Namely, we consider the case we have discussed in $\$ 3$ with $p=q=n$. Note in particular that $\omega$ can be written as

$$
\omega=\sum_{j=1}^{n}\left(\mathrm{~d} x_{j} \wedge \mathrm{d} y_{j}-\mathrm{d} x_{\bar{J}} \wedge \mathrm{d} y_{\bar{J}}\right)
$$

at $v=x+\mathrm{i} y \in W$ with $x={ }^{t}\left(x_{1}, \ldots, x_{2 n}\right), y={ }^{t}\left(y_{1}, \ldots, y_{2 n}\right) \in \mathbb{R}^{2 n}$. Then $(W, \omega)$ is a symplectic $G$-vector space, as above.

Remarks 4.1. (i) There is another realization of the Lie group $\mathrm{O}^{*}(2 n)$ as a group consisting of the complex orthogonal matrices. Namely,

$$
\mathrm{O}^{*}(2 n)=\left\{g \in \mathrm{GL}_{2 n} ;{ }^{t} g g=1,{ }^{t} g J_{n} g=J_{n}\right\}
$$

we temporarily denote this realization of $\mathrm{O}^{*}(2 n)$ by $G^{\gamma}$, because the former realization $G$ is isomorphic to $G^{\gamma}$ by the correspondence $G \ni g \mapsto \gamma g \gamma^{-1} \in G^{\gamma}$ with $\gamma=\frac{1}{\sqrt{2}}\left[\begin{array}{cc}1 & 1 \\ i & -i\end{array}\right] \in$ $\mathrm{U}(2 n)$ (cf. [Hel78]).

Let us consider the quaternionic vector space

$$
\mathbb{H}^{n}:=\left\{v={ }^{t}\left(v_{1}, \ldots, v_{n}\right) ; v_{i} \in \mathbb{H}(i=1, \ldots, n)\right\},
$$

where $\mathbb{H}=\{a+b \mathbf{i}+c \mathbf{j}+d \mathbf{k} ; a, b, c, d \in \mathbb{R}\}$ denotes the skew-field of quaternions. We regard $\mathbb{H}^{n}$ as a right $\mathbb{H}$-vector space. If we identify $\mathbf{i} \in \mathbb{H}$ with $\mathrm{i} \in \mathbb{C}$, then $\mathbb{H}^{n}$ is isomorphic to $\mathbb{C}^{2 n}$ by the map

$$
\phi_{1}: \mathbb{H}^{n} \rightarrow \mathbb{C}^{2 n}, \quad v=v^{\prime}+\mathbf{j} v^{\prime \prime} \mapsto\left[\begin{array}{c}
v^{\prime} \\
v^{\prime \prime}
\end{array}\right] \quad\left(v^{\prime}, v^{\prime \prime} \in \mathbb{C}^{n}\right),
$$

which is in fact a $\mathbb{C}$-isomorphism. Then $G^{\gamma}$ is characterized as the group consisting of $\mathbb{H}-$-linear transformations on $\mathbb{H}^{n}$ that preserve the quaternionic skew-Hermitian form $C$ given by

(see [GW10] for details).

$$
C(u, v):=u^{*} \mathbf{j} v \quad\left(u, v \in \mathbb{H}^{n}\right)
$$

(ii) There is another identification of $\mathbb{H}^{n}$ with a $\mathbb{C}$-vector space. Namely, there is an isomorphism of $\mathbb{H} \mathbb{H}^{n}$ onto $\mathrm{Mat}_{n \times 2}(\mathbb{C})$ given by

$$
\phi_{2}: \mathbb{H}^{n} \rightarrow \operatorname{Mat}_{n \times 2}(\mathbb{C}), \quad v=v^{\prime}+v^{\prime \prime} \mathbf{j} \mapsto\left[v^{\prime}, v^{\prime \prime}\right] .
$$

In this case, however, $\mathbb{H}^{n}$ is regarded as a left $\mathbb{H}$-vector space, and the map $\phi_{2}$ is a $\mathbb{C}$-isomorphism in this sense. Since $\mathbf{j} v^{\prime \prime}=\bar{v}^{\prime \prime} \mathbf{j}$ for $v^{\prime \prime} \in \mathbb{C}^{n}$, one sees that

$$
\left(\phi_{2} \circ \phi_{1}^{-1}\right)\left(\left[\begin{array}{c}
v^{\prime} \\
v^{\prime \prime}
\end{array}\right]\right)=\left[v^{\prime}, \bar{v}^{\prime \prime}\right] \text {. }
$$

Note that $\phi_{2} \circ \phi_{1}^{-1}$ is an $\mathbb{R}$-isomorphism from $\mathbb{C}^{2 n}$ onto $\operatorname{Mat}_{n \times 2}(\mathbb{C})$.

More generally, let us consider $\left(\mathbb{H} \mathbb{H}^{n}\right)^{k}$, the direct sum of $k$ copies of $\mathbb{H}^{n}$, which we regard as a left $\mathbb{H}\left[\right.$-vector space as above. Then the multiplication on $\left(\mathbb{H}^{n}\right)^{k}$ on the right by an element of $\operatorname{Mat}_{k}(\mathbb{H})$, say, $a+b \mathbf{j}$ with $a, b \in \operatorname{Mat}_{k}(\mathbb{C})$, corresponds to the multiplication on $\operatorname{Mat}_{n \times 2 k}(\mathbb{C})$ on the right by the complex $2 k \times 2 k$-matrix $\left[\begin{array}{cc}a & b \\ -\bar{b} & \bar{a}\end{array}\right]$. 
Lemma 4.2. The vector fields on $W$ generated by the basis (4.1) for $\mathfrak{g}_{0}=\mathfrak{v}^{*}(2 n)$ in the sense of (1.4) are given by

$$
\begin{aligned}
& \left(X_{i, j}^{c}\right)_{W}=-x_{j} \partial_{x_{i}}-y_{j} \partial_{y_{i}}+x_{i} \partial_{x_{j}}+y_{i} \partial_{y_{j}}-x_{\bar{J}} \partial_{x_{\bar{i}}}-y_{\bar{J}} \partial_{y_{\bar{i}}}+x_{\bar{l}} \partial_{x_{\bar{j}}}+y_{\bar{\imath}} \partial_{y_{\bar{j}}}, \\
& \left(Y_{i, j}^{c}\right)_{W}=y_{j} \partial_{x_{i}}+y_{i} \partial_{x_{j}}-y_{\bar{j}} \partial_{x_{\bar{i}}}-y_{\bar{\imath}} \partial_{x_{\bar{j}}}-x_{j} \partial_{y_{i}}-x_{i} \partial_{y_{j}}+x_{\bar{j}} \partial_{y_{\bar{l}}}+x_{\bar{l}} \partial_{y_{\bar{j}}} \text {, } \\
& \left(X_{i, j}^{n}\right)_{W}=-x_{\bar{J}} \partial_{x_{i}}+x_{\bar{\imath}} \partial_{x_{j}}+x_{j} \partial_{x_{\bar{i}}}-x_{i} \partial_{x_{\bar{j}}}-y_{\bar{j}} \partial_{y_{i}}+y_{\bar{\imath}} \partial_{y_{j}}+y_{j} \partial_{y_{\bar{i}}}-y_{i} \partial_{y_{\bar{j}}} \text {, } \\
& \left(Y_{i, j}^{n}\right)_{W}=y_{\bar{j}} \partial_{x_{i}}-y_{\bar{\imath}} \partial_{x_{j}}+y_{j} \partial_{x_{\bar{i}}}-y_{i} \partial_{x_{\bar{J}}}-x_{\bar{j}} \partial_{y_{i}}+x_{\bar{l}} \partial_{y_{j}}-x_{j} \partial_{y_{\bar{l}}}+x_{i} \partial_{y_{\bar{j}}} \text {. }
\end{aligned}
$$

For a given $v=\left[\begin{array}{l}v^{\prime} \\ v^{\prime \prime}\end{array}\right] \in \mathbb{C}^{2 n}$ with $v^{\prime}, v^{\prime \prime} \in \mathbb{C}^{n}$, we set $v_{+}:=\left(\phi_{2} \circ \phi_{1}^{-1}\right)(v)=\left[v^{\prime}, \bar{v}^{\prime \prime}\right] \in$ $\operatorname{Mat}_{n \times 2}(\mathbb{C})$ for brevity. By Remarks 4.1 (ii), $\operatorname{Sp}(1)$ acts on $W$ on the right via the $\mathbb{R}$ isomorphism $\phi_{2} \circ \phi_{1}^{-1}$.

Proposition 4.3. Let $(W, \omega)$ be as above and $G=\mathrm{O}^{*}(2 n)$. Then the moment map $\mu: W \rightarrow \mathfrak{g}_{0}^{*} \simeq \mathfrak{g}_{0}$ is given by

$$
\begin{aligned}
\mu(v) & =-\frac{\mathrm{i}}{2}\left(v v^{*} I_{n, n}-S^{t}\left(v v^{*} I_{n, n}\right) S\right) \\
& =-\frac{\mathrm{i}}{2}\left[\begin{array}{cc}
v_{+} v_{+}^{*} & -v_{+} J_{1}{ }^{t} v_{+} \\
-\bar{v}_{+} J_{1} v_{+}^{*} & -\bar{v}_{+}{ }^{t} v_{+}
\end{array}\right]
\end{aligned}
$$

for $v=x+\mathrm{i} y \in W$ with $x={ }^{t}\left(x_{1}, \ldots, x_{2 n}\right), y={ }^{t}\left(y_{1}, \ldots, y_{2 n}\right) \in \mathbb{R}^{2 n}$. In particular, $\mu$ is $G$-equivariant and is $\mathrm{Sp}(1)$-invariant.

Proof. It follows from Lemma4.2 that

$$
\langle\mu, X\rangle=\left\{\begin{array}{lll}
x_{i} y_{j}-x_{j} y_{i}-x_{\bar{l}} y_{\bar{J}}+x_{\bar{J}} y_{\bar{l}} & \text { if } & X=X_{i, j}^{c} \\
x_{i} x_{j}+x_{\bar{l}} x_{\bar{J}}+y_{i} y_{j}+y_{\bar{l}} y_{\bar{j}} & \text { if } & X=Y_{i, j}^{c} \\
x_{i} y_{\bar{J}}-x_{j} y_{\bar{l}}+x_{\bar{l}} y_{j}-x_{\bar{J}} y_{i} & \text { if } & X=X_{i, j}^{n} \\
x_{i} x_{\bar{J}}-x_{j} x_{\bar{l}}-y_{j} y_{\bar{l}}+y_{i} y_{\bar{J}} & \text { if } & X=Y_{i, j}^{n}
\end{array}\right.
$$

which can be rewritten in terms of complex coordinates defined by $z_{i}:=x_{i}+\mathrm{i} y_{i}, i=$ $1, \ldots, 2 n$, and their complex conjugates as

$$
\langle\mu, X\rangle=\left\{\begin{array}{rll}
-\frac{\mathrm{i}}{2}\left(\bar{z}_{i} z_{j}-\bar{z}_{j} z_{i}-\bar{z}_{\bar{l}} z_{\bar{j}}+\bar{z}_{\bar{j}} z_{\bar{l}}\right) & \text { if } & X=X_{i, j}^{c} ; \\
\frac{1}{2}\left(\bar{z}_{i} z_{j}+\bar{z}_{j} z_{i}+\bar{z}_{\bar{l}} z_{\bar{j}}+\bar{z}_{\bar{j}} \bar{z}_{\bar{l}}\right) & \text { if } & X=Y_{i, j}^{c} \\
-\frac{1}{2}\left(\bar{z}_{i} z_{\bar{J}}-\bar{z}_{j} z_{\bar{l}}+\bar{z}_{\bar{l}} z_{j}-\bar{z}_{\bar{j}} z_{i}\right) & \text { if } & X=X_{i, j}^{n} \\
\frac{1}{2}\left(\bar{z}_{i} z_{\bar{j}}-\bar{z}_{j} z_{\bar{l}}-\bar{z}_{\bar{l}} z_{j}+\bar{z}_{\bar{j}} z_{i}\right) & \text { if } & X=Y_{i, j}^{n} .
\end{array}\right.
$$

Thus, setting $v^{\prime}:={ }^{t}\left(z_{1}, \ldots, z_{n}\right)$ and $v^{\prime \prime}:={ }^{t}\left(z_{\overline{1}}, \ldots, z_{\bar{n}}\right)$, one obtains that

$$
\begin{aligned}
\mu(v) & =\sum_{i<j}\left\langle\mu, X_{i, j}^{c}\right\rangle\left(X_{i, j}^{c}\right)^{\vee}+\sum_{i \leqslant j}\left\langle\mu, Y_{i, j}^{c}\right\rangle\left(Y_{i, j}^{c}\right)^{\vee}+\sum_{i<j}\left\langle\mu, X_{i, j}^{n}\right\rangle\left(X_{i, j}^{n}\right)^{\vee}+\sum_{i<j}\left\langle\mu, Y_{i, j}^{n}\right\rangle\left(Y_{i, j}^{n}\right)^{\vee} \\
& =-\frac{\mathrm{i}}{2} \sum_{i, j=1}^{n}\left(\left(z_{i} \bar{z}_{j}+\bar{z}_{\bar{l}} z_{\bar{j}}\right) E_{i, j}-\left(\bar{z}_{i} z_{j}+z_{\bar{l}} \bar{z}_{\bar{j}}\right) E_{\bar{l}, \bar{j}}-\left(z_{i} \bar{z}_{\bar{j}}-\bar{z}_{\bar{l}} z_{j}\right) E_{i, \bar{j}}-\left(\bar{z}_{i} z_{\bar{j}}-z_{\bar{l}} \bar{z}_{j}\right) E_{\bar{l}, j}\right) \\
& =-\frac{\mathrm{i}}{2}\left[\begin{array}{cc}
v^{\prime} \bar{v}^{\prime}+\bar{v}^{\prime \prime} v^{\prime \prime} & -v^{\prime} \bar{v}^{\prime \prime}+\bar{v}^{\prime \prime} v^{\prime} \\
-\bar{v}^{\prime} v^{\prime \prime}+v^{\prime \prime} \bar{v}^{\prime} & -\bar{v}^{\prime} v^{\prime}-v^{\prime \prime} \bar{v}^{\prime \prime}
\end{array}\right]=-\frac{\mathrm{i}}{2}\left(\left[\begin{array}{c}
v^{\prime} \\
v^{\prime \prime}
\end{array}\right]{ }^{t}\left(\bar{v}^{\prime},-\bar{v}^{\prime \prime}\right)+\left[\begin{array}{c}
\bar{v}^{\prime \prime} \\
-\bar{v}^{\prime}
\end{array}\right]{ }^{t}\left(v^{\prime \prime},-v^{\prime}\right)\right)
\end{aligned}
$$




$$
=-\frac{\mathrm{i}}{2}\left(v v^{*} I_{n, n}-S^{t}\left(v v^{*} I_{n, n}\right) S\right) .
$$

Rewriting (4.10a) one obtains the second expression (4.10b).

The $\mathrm{Sp}(1)$-invariance of $\mu$ immediately follows from (4.10b), and the $G$-equivariance can be verified in the following way. If $g \in G$, then

$$
\mu(g v)=-\frac{\mathrm{i}}{2}\left(g v v^{*} g^{*} I_{n, n}-S^{t}\left(g v v^{*} g^{*} I_{n, n}\right) S\right)=-\frac{\mathrm{i}}{2}\left(g v v^{*} I_{n, n} g^{-1}-S^{t}\left(g v v^{*} I_{n, n} g^{-1}\right) S\right)
$$

since $g^{*} I_{n, n}=I_{n, n} g^{-1}$. The second term in the brace of the right-hand side equals

$$
S^{t} g^{-1 t}\left(v v^{*} I_{n, n}\right)^{t} g S=g S^{t}\left(v v^{*} I_{n, n}\right) S g^{-1}
$$

since ${ }^{t} g S=S g^{-1}$. Thus,

$$
\mu(g v)=-\frac{\mathrm{i}}{2}\left(g v v^{*} I_{n, n} g^{-1}-g S^{t}\left(v v^{*} I_{n, n}\right) S g^{-1}\right)=\operatorname{Ad}(g) \mu(v) .
$$

This completes the proof.

It follows from (4.4) that the Poisson brackets among $x_{i}, y_{i}, i=1, \ldots, 2 n$, are given by

$$
\left\{x_{i}, y_{j}\right\}=-\delta_{i, j}, \quad\left\{x_{\bar{l}}, y_{j}\right\}=\delta_{i, j}
$$

for $i, j=1, \ldots, n$, and all other brackets vanish. In terms of complex coordinates $z_{j}=x_{j}+\mathrm{i} y_{j}$ for $j=1, \ldots, 2 n$ and their conjugates, it follows from (4.13) that the Poisson brackets among them are given by

$$
\left\{z_{i}, \bar{z}_{j}\right\}=\left\{\bar{z}_{\bar{\imath}}, z_{\bar{\jmath}}\right\}=2 \mathrm{i} \delta_{i, j}
$$

for $i, j=1, \ldots, n$ and all other brackets vanish, as in (3.9). In view of (4.14), we quantize them by assigning

$$
\begin{array}{ll}
\widehat{z}_{i}=z_{i}, & \widehat{\bar{z}}_{i}=-2 \partial_{z_{i}}, \\
\widehat{\bar{z}}_{\bar{l}}=\bar{z}_{\bar{l}}, & \widehat{\bar{z}}_{\bar{l}}=-2 \partial_{\bar{z}_{\bar{\imath}}}
\end{array}
$$

for $i=1, \ldots, n$ so that the nontrivial commutators among the quantized operators are given by

$$
\left[\widehat{z}_{i}, \widehat{\bar{z}}_{j}\right]=\left[\widehat{\bar{z}}_{\bar{l}}, \widehat{z}_{\bar{j}}\right]=2 \delta_{i, j}
$$

for $i, j=1, \ldots, n$.

Let $I$ denote a complex structure on $W$ defined by $e_{j} \mapsto$ i $e_{j}$ and i $e_{j} \mapsto-e_{j}$ for $j=1, \ldots, 2 n$. Under the identification $e_{j} \leftrightarrow \partial_{x_{j}}$ and i $e_{j} \leftrightarrow \partial_{y_{j}}$, the classical observables $z_{j}$ and $\bar{z}_{j}$ introduced above can be regarded as the coordinate functions on $W_{\mathbb{C}}$ with respect to the basis $\frac{1}{2}\left(e_{j}-\mathrm{i} I e_{j}\right)$ and $\frac{1}{2}\left(e_{j}+\mathrm{i} I e_{j}\right)$ respectively for $j=1, \ldots, 2 n$. Note that $\bar{z}_{j}$ is no longer the complex conjugate of $z_{j}$ since $x_{i}$ and $y_{i}$ are now complex functions. Then the quantization (4.15) corresponds to taking a complex Lagrangian subspace $V$ given by

$$
V=\left\langle\frac{1}{2}\left(e_{j}-\mathrm{i} I e_{j}\right), \frac{1}{2}\left(e_{\bar{J}}+\mathrm{i} I e_{\bar{J}}\right) ; j=1, \ldots, n\right\rangle_{\mathbb{C}} .
$$

For simplicity, we set $w_{j}:=\bar{z}_{\bar{j}}, j=1, \ldots, n$, and write an element of $V=\operatorname{Mat}_{n \times 2}(\mathbb{C})$ as $[z, w]$ with $z={ }^{t}\left(z_{1}, \ldots, z_{n}\right)$ and $w={ }^{t}\left(w_{1}, \ldots, w_{n}\right)$ in what follows. 
Now, we quantize the moment map $\mu$ according to (4.15) using its first expression (4.10a) and denote the quantized moment map by $\widehat{\mu}$ as follows:

$$
\begin{aligned}
\widehat{\mu} & =-\frac{\mathrm{i}}{2}\left(\left[\begin{array}{c}
\widehat{z}_{1} \\
\vdots \\
\widehat{\bar{z}}_{\bar{n}}
\end{array}\right]\left(\widehat{\bar{z}}_{1}, \ldots, \widehat{\bar{z}}_{\bar{n}}\right) I_{n, n}-S I_{n, n}\left[\begin{array}{c}
\widehat{\bar{z}}_{1} \\
\vdots \\
\hat{\bar{z}}_{\bar{n}}
\end{array}\right]\left(\widehat{z}_{1}, \ldots, \widehat{z}_{\bar{n}}\right) S\right) \\
& =-\frac{\mathrm{i}}{2}\left(\left[\begin{array}{c}
z \\
-2 \partial_{w}
\end{array}\right]\left(-2^{t} \partial_{z},{ }^{t} w\right) I_{n, n}-S I_{n, n}\left[\begin{array}{c}
-2 \partial_{z} \\
w
\end{array}\right]\left({ }^{t} z,-2^{t} \partial_{w}\right) S\right) \\
& =\mathrm{i}\left[\begin{array}{cc}
z^{t} \partial_{z}+w^{t} \partial_{w} & \frac{1}{2}\left(z^{t} w-w^{t} z\right) \\
2\left(\partial_{z}{ }^{t} \partial_{w}-\partial_{w}{ }^{t} \partial_{z}\right) & -\left(\partial_{w}{ }^{t} w+\partial_{z}{ }^{t} z\right)
\end{array}\right]
\end{aligned}
$$

where $z={ }^{t}\left(z_{1}, \ldots, z_{n}\right), w={ }^{t}\left(w_{1}, \ldots, w_{n}\right), \partial_{z}={ }^{t}\left(\partial_{z_{1}}, \ldots, \partial_{z_{n}}\right)$ and $\partial_{w}={ }^{t}\left(\partial_{w_{1}}, \ldots, \partial_{w_{n}}\right)$. The quantization of the second expression (4.10b), i.e.,

$$
\widehat{\mu}=-\frac{\mathrm{i}}{2}\left[\begin{array}{cc}
\widehat{v}_{+}{ }^{t} \widehat{\bar{v}}_{+} & -\widehat{v}_{+} J_{1} t \widehat{v}_{+} \\
-\widehat{\bar{v}}_{+} J_{1} t \widehat{\bar{v}}_{+} & -\widehat{\bar{v}}_{+} t \widehat{v}_{+}
\end{array}\right]
$$

produces the same result as (4.18b), where $\widehat{v}_{+}=[z, w]$ and $\widehat{\bar{v}}_{+}=\left[-2 \partial_{z},-2 \partial_{w}\right]$.

Let $\mathcal{P}(V)$ denote the algebra of complex coefficient polynomials on $V$, i.e., $\mathcal{P}(V)=$ $\mathbb{C}\left[z_{1}, \ldots, z_{n}, w_{1}, \ldots, w_{n}\right]$, and $\mathcal{P} \mathcal{D}(V)$ the ring of polynomial coefficient differential operators on $V$. Note that the complex symplectic group of rank one

$$
\mathrm{Sp}_{1}=\left\{g \in \mathrm{GL}_{2} ;{ }^{t} g J_{1} g=J_{1}\right\}
$$

acts on $V$ by matrix multiplication on the right, and hence on $\mathcal{P}(V)$ by right regular representation, which we denote by $\rho$, as in (2.14). The right-action of $\mathrm{Sp}_{1}$ on $V$ coincides with the one on $\mathrm{Mat}_{n \times 2}(\mathbb{C})$ mentioned in Remarks 4.1(ii).

Theorem 4.4. For $X \in \mathfrak{g}=\mathfrak{v}_{2 n}$, set $\pi(X)=\mathrm{i}\langle\widehat{\mu}, X\rangle$. Then the map

$$
\pi: \mathfrak{g} \rightarrow \mathcal{P D}(V)
$$

is a Lie algebra homomorphism. In terms of the basis (4.3) for $\mathfrak{g}$, it is given by

$$
\pi(X)=\left\{\begin{array}{lll}
-\left(z_{j} \partial_{z_{i}}+w_{j} \partial_{w_{i}}+\delta_{i, j}\right) & \text { if } \quad X=X_{i, j}^{0} \\
2\left(\partial_{z_{i}} \partial_{w_{j}}-\partial_{w_{i}} \partial_{z_{j}}\right) & \text { if } \quad X=X_{i, j}^{+} \\
\frac{1}{2}\left(z_{j} w_{i}-w_{j} z_{i}\right) & \text { if } X=X_{i, j}^{-}
\end{array}\right.
$$

Moreover, $\pi(X)$ commutes with the action of $\mathrm{Sp}_{1}$ i.e., $\pi(X) \in \mathcal{P} \mathcal{D}(V)^{\mathrm{Sp}_{1}}$ for all $X \in \mathfrak{g}$.

Proof. It suffices to prove that $\pi(X)$ commutes with the right-action of $\mathrm{Sp}_{1}$. For this, we use the second expression (4.18c) of $\widehat{\mu}$. It follows from Lemma 2.6 that

$$
\operatorname{Ad}_{\rho(g)^{-1}} \widehat{v}_{+}=\widehat{v}_{+} g^{-1} \text { and } \quad \operatorname{Ad}_{\rho(g)^{-1}} \widehat{\bar{v}}_{+}=\widehat{\bar{v}}_{+}^{t} g
$$

for $g \in \mathrm{GL}_{2}$. Therefore, if $g \in \mathrm{Sp}_{1}$ then one obtains

$$
\operatorname{Ad}_{\rho(g)^{-1}} \widehat{\mu}=-\frac{\mathrm{i}}{2}\left[\begin{array}{cc}
\widehat{v}_{+} g^{-1}{ }^{t}\left(\widehat{\bar{v}}_{+}{ }^{t} g\right) & -\widehat{v}_{+} g^{-1} J_{1}{ }^{t}\left(\widehat{v}_{+} g^{-1}\right) \\
-\widehat{\bar{v}}_{+}{ }^{t} g J_{1}{ }^{t}\left(\widehat{\bar{v}}_{+}{ }^{t} g\right) & -\widehat{\bar{v}}_{+}{ }^{t} g^{t}\left(\widehat{v}_{+} g^{-1}\right)
\end{array}\right]
$$




$$
=-\frac{\mathrm{i}}{2}\left[\begin{array}{cc}
\widehat{v}_{+}{ }^{t} \widehat{\bar{v}}_{+} & -\widehat{v}_{+} J_{1}{ }^{t} \widehat{v}_{+} \\
-\widehat{\bar{v}}_{+} J_{1}{ }^{t} \widehat{\bar{v}}_{+} & -\widehat{\bar{v}}_{+}{ }^{t} \widehat{v}_{+}
\end{array}\right]=\widehat{\mu}
$$

since ${ }^{t} g J_{1} g=J_{1}$. This completes the proof.

4.2. Now let us consider $W^{k}$, the direct sum of $k$ copies of $W=\left(\mathbb{C}^{2 n}\right)_{\mathbb{R}}$, which we identify with $\mathrm{Mat}_{2 n \times k}(\mathbb{C})$. It is equipped with a symplectic form given by

$$
\omega_{k}(u, v)=\operatorname{Im} \operatorname{tr}\left(u^{*} I_{n, n} v\right) \quad\left(u, v \in W^{k}\right),
$$

and is still acted on by $G=\mathrm{O}^{*}(2 n)$ symplectically by matrix multiplication on the left. Under the identification of $e_{i, a} \leftrightarrow \partial_{x_{i, a}}$ and i $e_{i, a} \leftrightarrow \partial_{y_{i, a}}$, we write an element of $W^{k}$ as $v={ }^{t}\left[v_{1}, \ldots, v_{2 n}\right]$, where $v_{i}=x_{i}+\mathrm{i} y_{i}$ are complex row vectors with $x_{i}=\left(x_{i, 1}, \ldots, x_{i, k}\right)$ and $y_{i}=\left(y_{i, 1}, \ldots, y_{i, k}\right)$ being real row vectors of size $k$ for $i=1, \ldots, 2 n$. Then $\omega_{k}$ is given by

$$
\omega_{k}=\sum_{1 \leqslant i \leqslant n, 1 \leqslant a \leqslant k}\left(\mathrm{~d} x_{i, a} \wedge \mathrm{d} y_{i, a}-\mathrm{d} x_{\bar{l}, a} \wedge \mathrm{d} y_{\bar{l}, a}\right)
$$

at $v={ }^{t}\left[v_{1}, \ldots, v_{2 n}\right] \in \operatorname{Mat}_{2 n \times k}(\mathbb{C})$. Moreover, the isomorphisms $\phi_{1}$ and $\phi_{2}$ defined by (4.5) and (4.7) respectively naturally extend to the one between $\left(\mathbb{H}^{n}\right)^{k}$ and $\operatorname{Mat}_{2 n \times k}(\mathbb{C})$ and the one between $\left(\mathbb{H}^{n}\right)^{k}$ and $\mathrm{Mat}_{n \times 2 k}(\mathbb{C})$ respectively, which we denote by the same symbols. Then $\operatorname{Sp}(k)$ acts on $W^{k}$ on the right via the $\mathbb{R}$-isomorphism $\phi_{2} \circ \phi_{1}^{-1}$, as above.

Proposition 4.5. Let $\left(W^{k}, \omega_{k}\right)$ be the symplectic $G$-vector space as above. Then the moment map $\mu: W^{k} \rightarrow \mathfrak{g}_{0}^{*} \simeq \mathfrak{g}_{0}$ is given by the same formulae as 4.10). Namely, for $v={ }^{t}\left[v^{\prime}, v^{\prime \prime}\right] \in W^{k}$ with $v^{\prime}, v^{\prime \prime} \in \operatorname{Mat}_{n \times k}(\mathbb{C})$,

$$
\begin{aligned}
\mu(v) & =-\frac{\mathrm{i}}{2}\left(v v^{*} I_{n, n}-S^{t}\left(v v^{*} I_{n, n}\right) S\right) \\
& =-\frac{\mathrm{i}}{2}\left[\begin{array}{cc}
v_{+} v_{+}^{*} & -v_{+} J_{k}{ }^{t} v_{+} \\
-\bar{v}_{+} J_{k} v_{+}^{*} & -\bar{v}_{+}{ }^{t} v_{+}
\end{array}\right],
\end{aligned}
$$

where $v_{+}=\left(\phi_{2} \circ \phi_{1}^{-1}\right)(v) \in \operatorname{Mat}_{n \times 2 k}(\mathbb{C})$. In particular, $\mu$ is G-equivariant and is $\operatorname{Sp}(k)$ invariant.

Proof. The vector fields on $W^{k}$ generated by the basis for $\mathfrak{g}_{0}$ are given by the same formulae as (4.9) in Lemma 4.2, with the understanding that $x_{i}, y_{i}, \partial_{x_{i}}$ and $\partial_{y_{i}}$ are row vectors and the products stand for the inner product of row vectors. Now, exactly the same argument as in Proposition 4.3 implies the proposition.

It follows from (4.20) that the Poisson brackets among the real coordinate functions $x_{i, a}, y_{i, a}$ are given by

$$
\left\{x_{i, a}, y_{j, b}\right\}=-\delta_{i, j} \delta_{a, b}, \quad\left\{x_{\bar{\imath}, a}, y_{\bar{j}, b}\right\}=\delta_{i, j} \delta_{a, b}
$$

and all other brackets vanish, and hence the nontrivial ones among the complex coordinate functions are given by

$$
\left\{z_{i, a}, \bar{z}_{j, b}\right\}=\left\{\bar{z}_{\bar{l}, a}, z_{\bar{\jmath}, b}\right\}=2 \mathrm{i} \delta_{i, j} \delta_{a, b}
$$

for $i, j=1, \ldots, n$ and $a, b=1, \ldots, k$. Therefore, we quantize $z_{i, a}$ and $\bar{z}_{i, a}$ by assigning

$$
\begin{array}{ll}
\widehat{z}_{i, a}=z_{i, a}, & \widehat{\bar{z}}_{i, a}=-2 \partial_{z_{i, a}}, \\
\widehat{\bar{z}}_{\bar{l}, a}=\bar{z}_{\bar{i}, a}, & \widehat{\bar{z}}_{\bar{l}, a}=-2 \partial_{\bar{z}_{\bar{i}, a}}
\end{array}
$$


so that the nontrivial commutators among the quantized operators are given by

$$
\left[\widehat{z}_{i, a}, \widehat{\bar{z}}_{j, b}\right]=\left[\widehat{\bar{z}}_{\bar{i}, a}, \widehat{z}_{\bar{j}, b}\right]=2 \delta_{i, j} \delta_{a, b}
$$

for $i, j=1, \ldots, n$ and $a, b=1, \ldots, k$.

Let $V^{k}$ denote the direct sum of $k$ copies of $V$, with $V$ as in (4.17). Since $V^{k}$ can be identified with $\operatorname{Mat}_{n \times 2 k}(\mathbb{C})$, we write an element of $V^{k}$ as $[z, w]$, where $z=\left(z_{i, a}\right)$ and $w=\left(w_{i, a}\right)$ are elements of $\operatorname{Mat}_{n \times k}(\mathbb{C})$, and we set $w_{i, a}=\bar{z}_{\bar{l}, a}$ for $i=1, \ldots, n$ and $a=1, \ldots, k$ for simplicity, as above. Let $\mathcal{P}\left(V^{k}\right)=\mathbb{C}\left[z_{i, a}, w_{i, a} ; i=1, \ldots, n, a=1, \ldots, k\right]$ be the algebra of complex polynomial functions on $V^{k}$, and $\mathcal{P} \mathcal{D}\left(V^{k}\right)$ the ring of polynomial coefficient differential operators on $V^{k}$. Then the complex symplectic group $\mathrm{Sp}_{k}$ acts on $V^{k}$ by matrix multiplication on the right, and hence on $\mathcal{P}\left(V^{k}\right)$ by right regular representation, which we denote by $\rho$, as usual.

The quantized moment map $\widehat{\mu}$ is given by the same formula as $4.18 \mathrm{~b}$ ):

$$
\widehat{\mu}=\mathrm{i}\left[\begin{array}{cc}
z^{t} \partial_{z}+w^{t} \partial_{w} & \frac{1}{2}\left(z^{t} w-w^{t} z\right) \\
2\left(\partial_{z}{ }^{t} \partial_{w}-\partial_{w}{ }^{t} \partial_{z}\right) & -\left(\partial_{w}{ }^{t} w+\partial_{z}{ }^{t} z\right)
\end{array}\right] .
$$

Here, $z$ (resp. $w$ ) and $\partial_{z}$ (resp. $\partial_{w}$ ) now denote $n \times k$-matrices whose $(i, a)$-th entries are the multiplication operator $z_{i, a}\left(\right.$ resp. $\left.w_{i, a}\right)$ and the differential operator $\partial_{z_{i, a}}$ (resp. $\partial_{w_{i, a}}$ ) for $i=1, \ldots, n$ and $a=1, \ldots, k$.

Corollary 4.6. For $X \in \mathfrak{g}=\mathfrak{v}_{2 n}$, set $\pi(X)=\mathrm{i}\langle\widehat{\mu}, X\rangle$. Then the map

$$
\pi: \mathfrak{g} \rightarrow \mathcal{P D}\left(V^{k}\right)
$$

is a Lie algebra homomorphism. In terms of the basis (4.3) for $\mathfrak{g}$, it is given by

$$
\pi(X)=\left\{\begin{array}{lll}
-\sum_{a=1}^{k}\left(z_{j, a} \partial_{z_{i, a}}+w_{j, a} \partial_{w_{i, a}}+k \delta_{i, j}\right) & \text { if } \quad X=X_{i, j}^{0} \\
2 \sum_{a=1}^{k}\left(\partial_{z_{i, a}} \partial_{w_{j, a}}-\partial_{w_{i, a}} \partial_{z_{j, a}}\right) & \text { if } \quad X=X_{i, j}^{+} \\
\frac{1}{2} \sum_{a=1}^{k}\left(z_{j, a} w_{i, a}-w_{j, a} z_{i, a}\right) & \text { if } \quad X=X_{i, j}^{-}
\end{array}\right.
$$

Moreover, $\pi(X)$ commutes with the action of the complex symplectic group $\mathrm{Sp}_{k}$, i.e., $\pi(X) \in \mathcal{P D}\left(V^{k}\right)^{\mathrm{Sp}_{k}}$ for all $X \in \mathfrak{g}$.

Proof. The proof is essentially the same as that of Theorem 4.4 .

Similarly to the cases discussed above, it is well known that the irreducible decomposition of $\mathcal{P}\left(V^{k}\right)$ under the joint action of $\left(\mathfrak{b}_{2 n}, \mathrm{Sp}_{k}\right)$ is given by

$$
\mathcal{P}\left(V^{k}\right) \simeq \sum_{\sigma \in \widehat{\mathrm{Sp}_{k}}, L(\sigma) \neq\{0\}} L(\sigma) \otimes V_{\sigma},
$$

where $V_{\sigma}$ is a representative of the class $\sigma \in \widehat{\operatorname{Sp}}_{k}$, the set of all equivalence classes of the finite-dimensional irreducible representation of $\mathrm{Sp}_{k}$, and $L(\sigma):=\operatorname{Hom}_{\mathrm{Sp}_{k}}\left(V_{\sigma}, \mathcal{P}\left(V^{k}\right)\right)$ which is an infinite-dimensional irreducible representation of $\mathfrak{p}_{2 n}$. It is also well known that the action $\pi$ restricted to ${ }^{\prime}$ lifts to the complexification $K_{\mathbb{C}}$ of the maximal compact subgroup $K$ of $G=\mathrm{O}^{*}(2 n)$, which implies that $L(\sigma)$ is an irreducible $\left(\mathfrak{g}, K_{\mathbb{C}}\right)$-module. 


\section{LAGRANGIAN SUBSPACE}

In this section, we take complex Lagrangian subspaces of $W_{\mathbb{C}}$ different from the ones considered in the previous sections in the cases where $G=\mathrm{O}^{*}(2 n)$ and $\mathrm{U}(p, q)$, and quantize the moment map to obtain finite-dimensional representations of $2 n$ and the oscillator representation of $\mathfrak{u}(p, q)$. Finally, we make an observation that the image of the Lagrangian subspace coincides with the associated variety of the corresponding irreducible $\left(\mathfrak{g}, K_{\mathbb{C}}\right)$ (or $\left(\mathfrak{g}, \tilde{K}_{\mathbb{C}}\right)$ )-modules occurring in the irreducible decomposition of the space consisting of polynomial functions on the Lagrangian subspace under the joint action of $\left(\mathfrak{g}, G^{\prime}\right)$.

5.1. Let $G=\mathrm{O}^{*}(2 n)$ and let $(W, \omega)$ be the symplectic $G$-vector space we discussed in $\$ 4$, i.e., $W=\left(\mathbb{C}^{2 n}\right)_{\mathbb{R}}$ and $\omega$ is given by (4.4). Let us now consider another complex Lagrangian subspace $V^{\prime} \subset W_{\mathbb{C}}$ defined by

$$
V^{\prime}:=\left\langle\frac{1}{2}\left(e_{1}-\mathrm{i} I e_{1}\right), \ldots, \frac{1}{2}\left(e_{2 n}-\mathrm{i} I e_{2 n}\right)\right\rangle_{\mathbb{C}}
$$

and the corresponding quantization

$$
\widehat{z}_{i}=z_{i}, \quad \widehat{\bar{z}}_{i}=-2 \epsilon_{i} \partial_{z_{i}}
$$

for $i=1, \ldots, 2 n$ as in $\$ 3$, which also satisfy (4.25). Here $I$ denotes the complex structure on $W$ mentioned in $\$ 4$ Then the quantized moment map, which we denote by the same symbol $\widehat{\mu}$, is given by

$$
\begin{aligned}
& \widehat{\mu}=-\frac{\mathrm{i}}{2}\left(\left[\begin{array}{c}
\widehat{z}_{1} \\
\vdots \\
\widehat{\bar{z}}_{\bar{n}}
\end{array}\right]\left(\widehat{\bar{z}}_{1}, \ldots, \widehat{\bar{z}}_{\bar{n}}\right) I_{n, n}-S I_{n, n}\left[\begin{array}{c}
\widehat{\bar{z}}_{1} \\
\vdots \\
\widehat{\bar{z}}_{\bar{n}}
\end{array}\right]\left(\widehat{z}_{1}, \ldots, \widehat{\bar{z}}_{\bar{n}}\right) S\right) \\
& =-\mathrm{i}\left[\begin{array}{ll}
-z^{\prime} \partial_{z^{\prime}}+\partial_{z^{\prime \prime}}{ }^{t} z^{\prime \prime} & -z^{\prime} \partial_{z^{\prime \prime}}+\partial_{z^{\prime \prime}}{ }^{t} z^{\prime} \\
-z^{\prime \prime}{ }^{t} \partial_{z^{\prime}}+\partial_{z^{\prime}}{ }^{t} z^{\prime \prime} & -z^{\prime \prime} \partial_{z^{\prime \prime}}+\partial_{z^{\prime}} z^{\prime}
\end{array}\right]
\end{aligned}
$$

with $z^{\prime}={ }^{t}\left(z_{1}, \ldots, z_{n}\right), z^{\prime \prime}={ }^{t}\left(z_{\overline{1}}, \ldots, z_{\bar{n}}\right), \partial_{z^{\prime}}={ }^{t}\left(\partial_{z_{1}}, \ldots, \partial_{z_{n}}\right)$ and $\partial_{z^{\prime \prime}}={ }^{t}\left(\partial_{z_{\overline{1}}}, \ldots, \partial_{z_{\bar{n}}}\right)$. Therefore, in terms of the basis (4.3) for $\mathfrak{g}=\mathfrak{v}_{2 n}, \pi(X):=\mathrm{i}\langle\hat{\mu}, X\rangle$ is given by

$$
\pi(X)=\left\{\begin{array}{llc}
z_{j} \partial_{z_{i}}-z_{\bar{\imath}} \partial_{z_{\bar{j}}} & \text { if } \quad X=X_{i, j}^{0} \\
z_{\bar{J}} \partial_{z_{i}}-z_{\bar{l}} \partial_{z_{j}} & \text { if } \quad X=X_{i, j}^{+} \\
z_{i} \partial_{z_{\bar{j}}}-z_{j} \partial_{z_{\bar{\imath}}} & \text { if } \quad X=X_{i, j}^{-}
\end{array}\right.
$$

Since each $\pi(X)$ preserves the degree of a homogeneous polynomial $f \in \mathcal{P}\left(V^{\prime}\right)=$ $\mathbb{C}\left[z_{1}, \ldots, z_{2 n}\right]$ for $X \in \mathfrak{g}$, any irreducible representation occurring in the irreducible decomposition of $\mathcal{P}\left(V^{\prime}\right)$ is finite-dimensional.

5.2. On the contrary, we will apply the quantization procedure introduced in $\$ 4$ to the case discussed in $\$ 3$. Namely, let $G=\mathrm{U}(p, q)$ and let $(W, \omega)$ be the symplectic $G$ vector space, i.e., $W=\left(\mathbb{C}^{p+q}\right)_{\mathbb{R}}$ and $\omega$ is given by (3.2). Now we quantize the complex coordinate functions $z_{j}=x_{j}+\mathrm{i} y_{j}$ and $\bar{z}_{j}=x_{j}-\mathrm{i} y_{j}$ in the following way (cf. (4.15)):

$$
\begin{aligned}
& \widehat{z}_{i}=z_{i}, \quad \widehat{\bar{z}}_{i}=-2 \partial_{z_{i}}, \quad(i=1, \ldots, p) ; \\
& \widehat{\bar{z}}_{\bar{J}}=\bar{z}_{\bar{j}}, \quad \widehat{z}_{\bar{J}}=-2 \partial_{\bar{z}_{\bar{j}}}, \quad(j=1, \ldots, q),
\end{aligned}
$$


which also satisfy (3.11). This quantization corresponds to taking a complex Lagrangian subspace $V \subset W_{\mathbb{C}}$ defined by

$$
V=\left\langle\frac{1}{2}\left(e_{i}-\mathrm{i} I e_{i}\right), \frac{1}{2}\left(e_{\bar{J}}+\mathrm{i} I e_{\bar{J}}\right) ; i=1, \ldots, p, j=1, \ldots, q\right\rangle_{\mathbb{C}},
$$

where $I$ denotes the complex structure on $W$ mentioned in $\$ 3$. For simplicity, we will write $w_{j}:=\bar{z}_{\bar{J}}, j=1, \ldots, q$, as in the previous section, and write an element of $V$ as $\left[\begin{array}{l}z \\ w\end{array}\right]$ with $z \in \mathbb{C}^{p}$ and $w \in \mathbb{C}^{q}$. Then the quantized moment map, which we denote by the same symbol $\widehat{\mu}$, is given by

$$
\begin{aligned}
\widehat{\mu} & =-\frac{\mathrm{i}}{2}\left[\begin{array}{c}
\widehat{z}_{1} \\
\vdots \\
\widehat{\bar{z}}_{n}
\end{array}\right]\left(\widehat{\bar{z}}_{1}, \ldots, \widehat{\bar{z}}_{n}\right) I_{p, q}=-\frac{\mathrm{i}}{2}\left[\begin{array}{c}
z \\
-2 \partial_{w}
\end{array}\right]\left(-2^{t} \partial_{z},-{ }^{t} w\right) \\
& =\mathrm{i}\left[\begin{array}{cc}
z^{t} \partial_{z} & \frac{1}{2} z^{t} w \\
-2 \partial_{w}{ }^{t} \partial_{z} & -\partial_{w}{ }^{t} w
\end{array}\right]
\end{aligned}
$$

with $z={ }^{t}\left(z_{1}, \ldots, z_{p}\right), \partial_{z}={ }^{t}\left(\partial_{z_{1}}, \ldots, \partial_{z_{p}}\right), w={ }^{t}\left(w_{1}, \ldots, w_{q}\right)$ and $\partial_{w}={ }^{t}\left(\partial_{w_{1}}, \ldots, \partial_{w_{q}}\right)$. In terms of the basis $\left\{E_{i, j}\right\}$ for $\mathfrak{g}=\mathfrak{g l}_{n}, \pi(X):=\mathrm{i}\langle\widehat{\mu}, X\rangle$ is given by

$$
\pi(X)=\left\{\begin{array}{clll}
-z_{j} \partial_{z_{i}} & \text { if } \quad X=E_{i, j} & (i, j=1, \ldots, p) \\
2 \partial_{z_{i}} \partial_{w_{j}} & \text { if } \quad X=E_{i, \bar{J}} & (i=1, \ldots, p ; j=1, \ldots, q) \\
-\frac{1}{2} z_{j} w_{i} & \text { if } \quad X=E_{\bar{l}, j} \quad(i=1, \ldots, q ; j=1, \ldots, p) \\
\partial_{w_{j}} w_{i} & \text { if } \quad X=E_{\bar{l}, \bar{j}} \quad(i, j=1, \ldots, q) .
\end{array}\right.
$$

Let us now consider the $k$ direct sum $W_{\mathbb{C}}^{k}$, and its subspace $V^{k}$ with $V$ given in (5.6) which is identified with $\operatorname{Mat}_{n \times k}(\mathbb{C})$. Then $\mathrm{GL}_{k}$ acts on $V^{k}$ on the right by

$$
\left[\begin{array}{c}
z \\
w
\end{array}\right] \mapsto\left[\begin{array}{c}
z g \\
w^{t} g^{-1}
\end{array}\right]
$$

for $g \in \mathrm{GL}_{k}$, with $z=\left(z_{i, a}\right) \in \operatorname{Mat}_{p \times k}(\mathbb{C})$ and $w=\left(w_{j, a}\right) \in \operatorname{Mat}_{q \times k}(\mathbb{C})$, and hence on $\mathcal{P}\left(V^{k}\right)$ by right regular representation, which we denote by $\rho$, as usual. Note that $(5.9)$ is the holomorphic extension of the standard right-action of $\mathrm{U}(k)$ on $\mathrm{Mat}_{n \times k}(\mathbb{C})$ given by $Z \mapsto Z g$ for $Z \in \mathrm{Mat}_{n \times k}(\mathbb{C})$ and $g \in \mathrm{U}(k)$. Then, understanding that $z$ and $\partial_{z}$ (resp. $w$ and $\left.\partial_{w}\right)$ in (5.7) stand for $p \times k$-matrices $\left(z_{i, a}\right)$ and $\left(\partial_{z_{i, a}}\right)\left(\right.$ resp. $q \times k$-matrices $\left(w_{j, a}\right)$ and $\left.\left(\partial_{w_{j, a}}\right)\right)$ as in the previous sections, one obtains the following.

Theorem 5.1. For $X \in \mathfrak{g}=\mathfrak{g l}_{n}$, set $\pi(X):=\mathrm{i}\langle\widehat{\mu}, X\rangle$. Then the map

$$
\pi: \mathfrak{g} \rightarrow \mathcal{P D}\left(V^{k}\right)
$$

is a Lie algebra homomorphism. Moreover, $\pi(X)$ commutes with the action of $\mathrm{GL}_{k}$ on $V^{k}$, i.e., $\pi(X) \in \mathcal{P D}\left(V^{k}\right)^{\mathrm{GL}_{k}}$ for all $X \in \mathfrak{g}$.

Proof. We only show that $\pi(X) \in \mathcal{P D}\left(V^{k}\right)^{\mathrm{GL}_{k}}$ for $X \in \mathfrak{g}$. It follows from Lemma2.6 that

$$
\begin{aligned}
\operatorname{Ad}_{\rho(g)^{-1}} z & =z g^{-1}, & \operatorname{Ad}_{\rho(g)^{-1}} w & =w^{t} g, \\
\operatorname{Ad}_{\rho(g)^{-1}} \partial_{z} & =\partial_{z}{ }^{t} g, & \operatorname{Ad}_{\rho(g)^{-1}} \partial_{w} & =\partial_{w} g^{-1}
\end{aligned}
$$


for $g \in \mathrm{GL}_{k}$. Hence one obtains that

$$
\begin{aligned}
\mathbf{A d}_{\rho(g)^{-1}} \widehat{\mu} & =-\frac{\mathrm{i}}{2}\left[\begin{array}{c}
\mathbf{A d}_{\rho(g)^{-1} z} \\
-2 \mathbf{A d}_{\rho(g)^{-1}} \partial_{w}
\end{array}\right]\left[-2^{t}\left(\mathbf{A d}_{\rho(g)^{-1}} \partial_{z}\right),{ }^{t}\left(\mathbf{A d}_{\left.\rho(g)^{-1} w\right)}\right]\right. \\
& =-\frac{\mathrm{i}}{2}\left[\begin{array}{c}
z g^{-1} \\
-2 \partial_{w} g^{-1}
\end{array}\right]\left[-2 g^{t} \partial_{z}, g^{t} w\right] \\
& =-\frac{\mathrm{i}}{2}\left[\begin{array}{c}
z \\
-2 \partial_{w}
\end{array}\right] g^{-1} g\left[-2^{t} \partial_{z},{ }^{t} w\right]=\widehat{\mu} .
\end{aligned}
$$

This completes the proof.

Therefore, the irreducible decomposition of $\mathcal{P}\left(V^{k}\right)$ is given by

$$
\mathcal{P}\left(V^{k}\right) \simeq \sum_{\sigma \in \widehat{\mathrm{GL}}_{k}, L(\sigma) \neq\{0\}} L(\sigma) \otimes V_{\sigma},
$$

where $V_{\sigma}$ is a representative of the class $\sigma \in \widehat{\mathrm{GL}}_{k}$, the set of all equivalence classes of the finite-dimensional irreducible representation of $\mathrm{GL}_{k}$, and $L(\sigma):=\operatorname{Hom}_{\mathrm{GL}_{k}}\left(V_{\sigma}, \mathcal{P}\left(V^{k}\right)\right.$ ). It is well known that $L(\sigma)$ is an irreducible $\left(\mathfrak{g}, K_{\mathbb{C}}\right)$-module of infinite dimension for any $\sigma \in \widehat{\mathrm{GL}}_{k}$ such that $L(\sigma) \neq\{0\}$, where $\mathfrak{g}=\mathfrak{g l}_{p+q}$ and $K_{\mathbb{C}}$ is the complexification of the maximal compact subgroup $K$ of $G=\mathrm{U}(p, q)$.

5.3. One can uniquely extend the moment map $\mu: W \rightarrow \mathfrak{g}_{0}$ to the map from $W_{\mathbb{C}}$ into $\mathfrak{g}$, which we denote by $\mu_{\mathbb{C}}$. Then the images by $\mu_{\mathbb{C}}$ of the complex Lagrangian subspaces that have been considered in this and previous sections are all equal to the associated varieties of the corresponding representations, which we will see below case by case.

5.3.1. First we consider the cases where $G=\mathrm{U}(p, q)$ and $\mathrm{O}^{*}(2 n)$. Let $K_{\mathbb{C}}$ be the complexification of the maximal compact group $K$ of $G$. Then it is well known that $K_{\mathbb{C}}$ acts on $\mathfrak{p}$ with the irreducible decomposition $\mathfrak{p}=\mathfrak{p}^{+} \oplus \mathfrak{p}^{-}$and that the orbit space decomposition of $\mathfrak{p}^{+}$under $K_{\mathbb{C}}$ is given by

$$
\mathfrak{p}^{+}=\bigsqcup_{j=0}^{r} \mathcal{O}_{j}^{K_{\mathbb{C}}} \quad(r:=\mathbb{R}-\operatorname{rank} G),
$$

where

$$
\begin{aligned}
& \mathcal{O}_{j}^{K_{\mathbb{C}}}=\left\{\left[\begin{array}{ll}
O & C \\
O & O
\end{array}\right] ; \quad C \in \mathrm{Mat}_{p \times q}(\mathbb{C}), \operatorname{rank} C=j\right\} \quad \text { for } G=\mathrm{U}(p, q), \\
& \mathcal{O}_{j}^{K_{\mathbb{C}}}=\left\{\left[\begin{array}{ll}
O & C \\
O & O
\end{array}\right] ; \begin{array}{l}
C \in \mathrm{Mat}_{n \times n}(\mathbb{C}),{ }^{t} C+C=O \\
\operatorname{rank} C=2 j
\end{array}\right\} \quad \text { for } G=\mathrm{O}^{*}(2 n) .
\end{aligned}
$$

Moreover, if we denote the closure of a orbit $\mathcal{O}$ by $\overline{\mathcal{O}}$, then

$$
\overline{\mathcal{O}_{j}^{K_{\mathbb{C}}}}=\bigsqcup_{i \leqslant j} \mathcal{O}_{i}^{K_{\mathbb{C}}}
$$

(see [KR71]). Therefore, in view of the explicit formulae (3.5) and (4.10), one finds that

$$
\mu_{\mathbb{C}}\left(V^{\prime k}\right)=\overline{\mathcal{O}_{0}^{K_{\mathbb{C}}}}=\{0\} \quad \text { and } \quad \mu_{\mathbb{C}}\left(V^{k}\right)=\overline{\mathcal{O}_{m}^{K_{\mathbb{C}}}}
$$


with $m=\min (k, r)$. Since the associated varieties of the finite-dimensional representations and those of the irreducible representations $L(\sigma)$ occurring in (5.10) and (4.27) are equal to $\{0\}$ and $\overline{\mathcal{O}_{m}^{K_{\mathrm{C}}}}$ respectively (cf. [DES91]), one concludes that the image of the complex Lagrangian subspace $V^{k}$ or $V^{\prime k}$ by $\mu_{\mathbb{C}}$ coincides with the associated variety corresponding to the irreducible representations occurring in $\mathcal{P}\left(V^{k}\right)$ or in $\mathcal{P}\left(V^{\prime k}\right)$.

5.3.2. In order to see this is the case for $G=\operatorname{Sp}(n, \mathbb{R})$, we realize the symplectic group over $\mathbb{R}$ as its Cayley transform:

$$
G^{\gamma}:=\left\{\gamma g \gamma^{-1} ; g \in G\right\}=\mathrm{Sp}_{n} \cap \mathrm{U}(n, n)
$$

with $\gamma=\frac{1}{2}\left[\begin{array}{cc}1 & 1 \\ -\mathrm{i} & \mathrm{i}\end{array}\right]$. In the rest of this subsection, however, let us denote $G^{\gamma}$ just by $G$, and use the same symbols to denote the Cayley transforms of subgroups, Lie algebras etc. as those of the corresponding objects by abuse of notation if there is no risk of confusion.

One can also obtain the so-called Fock model of the oscillator representation by the canonical quantization of the moment map in the following way: let us denote by $I$ the complex structure on $W=\mathbb{R}^{2 n}$ defined by $e_{i} \mapsto e_{\bar{\imath}}$ and $e_{\bar{\imath}} \mapsto-e_{i}$ for $i=1, \ldots, n$, and introduce complex coordinates $z_{i}:=x_{i}+\mathrm{i} y_{i}$ and their conjugates $\bar{z}_{i}:=x_{i}-\mathrm{i} y_{i}$, $i=1, \ldots, n$. Namely, we regard $W=\mathbb{R}^{2 n}$ as $\left(\mathbb{C}^{n}\right)_{\mathbb{R}}$; more precisely, let us define an $\mathbb{R}$-vector space $W_{a}$ by $W_{a}=\left\{\left[\begin{array}{l}z \\ \bar{z}\end{array}\right] ; z \in \mathbb{C}^{n}\right\}$ and an $\mathbb{R}$-isomorphism from $W_{a}$ onto $W$ by

$$
\varphi_{\gamma}: W_{a} \rightarrow W, \quad\left[\begin{array}{l}
z \\
\bar{z}
\end{array}\right] \mapsto \gamma\left[\begin{array}{l}
z \\
\bar{z}
\end{array}\right]=\frac{1}{2}\left[\begin{array}{c}
z+\bar{z} \\
-\mathrm{i}(z-\bar{z})
\end{array}\right],
$$

for $z={ }^{t}\left(z_{1}, \ldots, z_{n}\right)$ and $\bar{z}={ }^{t}\left(\bar{z}_{1}, \ldots, \bar{z}_{n}\right) \in \mathbb{C}^{n}$. The Cayley transform $G$ acts on $W_{a}$ by $v \mapsto g v$ (matrix multiplication) for $g \in G$ and $v \in W_{a}$, with respect to which $\varphi_{\gamma}$ is equivariant. Moreover, one sees that $\varphi_{\gamma}^{*} \omega=\frac{\mathrm{i}}{2} \sum_{i=1}^{n} \mathrm{~d} z_{i} \wedge \mathrm{d} \bar{z}_{i}$ and that the moment map $\mu: W_{a} \rightarrow \mathrm{g}_{0}$ is given by

$$
\mu(v)=\frac{\mathrm{i}}{2} v^{t} v J_{n}=\frac{\mathrm{i}}{2}\left[\begin{array}{ll}
-z^{t} \bar{z} & z^{t} z \\
-\bar{z}^{t} \bar{z} & \bar{z}^{t} z
\end{array}\right]
$$

for $v={ }^{t}\left(z_{1}, \ldots, z_{n}, \bar{z}_{1}, \ldots, \bar{z}_{n}\right) \in W_{a}$.

Remark 5.2. If we temporarily distinguish the Cayley transform $\mathfrak{g}_{0}^{\gamma}$ from $\mathfrak{g}_{0}$ only in this remark, it is easily verified that the following diagram is commutative:

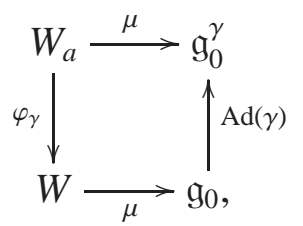

where the upper horizontal map denotes the moment map given by (5.16), while the lower horizontal one by (2.5).

The Poisson brackets among $z_{i}$ and $\bar{z}_{i}$ are given by (3.9) with all $\epsilon_{i}=1$. Therefore we quantize $z_{i}$ and $\bar{z}_{i}$ by assigning

$$
\widehat{z}_{i}=z_{i}, \quad \widehat{\bar{z}}_{i}=-2 \partial_{z_{i}},
$$


so that they satisfy (3.11) with all $\epsilon_{i}=1$. This quantization corresponds to the choice of the complex Lagrangian subspace $V$ of $W_{\mathbb{C}}=\mathbb{C}^{2 n}$ given by

$$
V=\left\langle\frac{1}{2}\left(e_{1}-\mathrm{i} e_{\overline{1}}\right), \ldots, \frac{1}{2}\left(e_{n}-\mathrm{i} e_{\bar{n}}\right)\right\rangle_{\mathbb{C}} .
$$

Then the quantized moment map, which we denote by $\widehat{\mu}$ as always, is given by

$$
\begin{aligned}
\widehat{\mu} & =\frac{\mathrm{i}}{2}\left[\begin{array}{c}
\widehat{z}_{1} \\
\vdots \\
\widehat{\bar{z}}_{n}
\end{array}\right]\left(\widehat{z}_{1}, \ldots, \widehat{\bar{z}}_{n}\right) J_{n}=\frac{\mathrm{i}}{2}\left[\begin{array}{c}
z \\
-2 \partial_{z}
\end{array}\right]\left({ }^{t} z,-2^{t} \partial_{z}\right) J_{n} \\
& =\mathrm{i}\left[\begin{array}{cc}
z^{t} \partial_{z} & \frac{1}{2} z^{t} z \\
-2 \partial_{z}{ }^{t} \partial_{z} & -\partial_{z}{ }^{t} z
\end{array}\right]
\end{aligned}
$$

with $z={ }^{t}\left(z_{1}, \ldots, z_{n}\right), \partial_{z}={ }^{t}\left(\partial_{z_{1}}, \ldots, \partial_{z_{n}}\right)$. In terms of the basis $\left\{X_{i, j}^{\star}\right\}$ for $\mathfrak{g}=\mathfrak{s p}_{n}$, $\pi(X):=\mathrm{i}\langle\widehat{\mu}, X\rangle$ is given by

$$
\pi(X)=\left\{\begin{array}{lll}
-\frac{1}{2}\left(z_{j} \partial_{z_{i}}+\partial_{z_{i}} z_{j}\right) & \text { if } & X=X_{i, j}^{0} \\
2 \partial_{z_{i}} \partial_{z_{j}} & \text { if } & X=X_{i, j}^{+} \\
-\frac{1}{2} z_{j} z_{i} & \text { if } & X=X_{i, j}^{-}
\end{array}\right.
$$

Now let us take the $k$ direct sum $W_{\mathbb{C}}^{k}$ and its subspace $V^{k}$, with $V$ as in (5.19). When $V^{k}$ is identified with $\mathrm{Mat}_{n \times k}(\mathbb{C}), \mathrm{GL}_{k}$ acts on $V^{k}$ on the right and hence on $\mathcal{P}\left(V^{k}\right)$ by right regular representation. Then, if one understands that $z$ and $\partial_{z}$ in (5.20) stand for $n \times k$-matrices $\left(z_{i, a}\right)$ and $\left(\partial_{z_{i, a}}\right)$ respectively and sets $\pi(X)=\mathrm{i}\langle\widehat{\mu}, X\rangle$ for $X \in \mathfrak{g}=\mathfrak{s p}_{n}$, one can show that the map $\pi: \mathfrak{g} \rightarrow \mathcal{P D}\left(V^{k}\right)$ is a Lie algebra homomorphism and that $\pi(X) \in \mathcal{P} \mathcal{D}\left(V^{k}\right)^{\mathrm{O}_{k}}$ for all $X \in \mathfrak{g}$. The irreducible decomposition of $\mathcal{P}\left(V^{k}\right)$ under the joint action of $\left(\mathfrak{s p}_{n}, \mathrm{O}_{k}\right)$ is of course the same as (2.18).

It is known that the orbit space decomposition under $K_{\mathbb{C}}$ of $\mathfrak{p}^{+}$is given by the same formula as (5.11) with

$$
\mathcal{O}_{j}^{K_{\mathbb{C}}}=\left\{\left[\begin{array}{ll}
O & C \\
O & O
\end{array}\right] ; \begin{array}{l}
C \in \mathrm{Mat}_{n \times n}(\mathbb{C}),{ }^{t} C=C \\
\operatorname{rank} C=j
\end{array}\right\},
$$

and its closure $\overline{\mathcal{O}_{j}^{K_{\mathrm{C}}}}$ is given by the same formula as (5.14) (see [KR71]). Therefore, in view of (5.16), one finds that

$$
\mu_{\mathbb{C}}\left(V^{k}\right)=\overline{\mathcal{O}_{m}^{K_{\mathbb{C}}}}
$$

with $m=\min (k, r)$. Hence the image of the complex Lagrangian subspace $V^{k}$ by $\mu_{\mathbb{C}}$ coincides with the associated variety corresponding to the irreducible representations occurring in $\mathcal{P}\left(V^{k}\right)$, as in the previous cases.

\section{REFERENCES}

[AY09] N. Abe and H. Yamashita, A note on Howe duality correspondence and isotropy representations for unitary lowest weight modules of $\operatorname{Mp}(n, \mathbf{R})$, J. Lie Theory 19 (2009), no. 4, 671-683. MR 2599006 (2011f:22017)

[BW97] Sean Bates and Alan Weinstein, Lectures on the geometry of quantization, Berkeley Mathematics Lecture Notes, vol. 8, American Mathematical Society, Providence, RI; Berkeley Center for Pure and Applied Mathematics, Berkeley, CA, 1997. MR 1806388 (2002f:53151) 
[CG97] Neil Chriss and Victor Ginzburg, Representation theory and complex geometry, Birkhäuser Boston, Inc., Boston, MA, 1997. MR 1433132 (98i:22021)

[DES91] Mark G. Davidson, Thomas J. Enright, and Ronald J. Stanke, Differential operators and highest weight representations, Mem. Amer. Math. Soc. 94 (1991), no. 455, iv+102. MR 1081660 (92c:22034)

[EHW83] Thomas Enright, Roger Howe, and Nolan Wallach, A classification of unitary highest weight modules, Representation theory of reductive groups (Park City, Utah, 1982), Progr. Math., vol. 40, Birkhäuser Boston, Boston, MA, 1983, pp. 97-143. MR 733809 (86c:22028)

[GW10] R. Goodman and N. W. Wallach, Symmetry, representations and invariants, Graduate Texts in Math., vol. 255, Springer Verlag, 2010.

[Has11] T. Hashimoto, On the principal symbols of $K_{\mathbb{C}}$-invariant differential operators on Hermitian symmetric spaces, J. Math. Soc. Japan 63 (2011), 837-869, arXiv:0804.4038 [math.RT].

[Hel78] S. Helgason, Differential geometry, Lie groups, and symmetric spaces, Pure and App. Math., vol. 80, Academic Press, 1978.

[HKM14] Joachim Hilgert, Toshiyuki Kobayashi, and Jan Möllers, Minimal representations via Bessel operators, J. Math. Soc. Japan 66 (2014), no. 2, 349-414. MR 3201818

[HKMØ12] Joachim Hilgert, Toshiyuki Kobayashi, Jan Möllers, and Bent Ørsted, Fock model and Segal-Bargmann transform for minimal representations of Hermitian Lie groups, J. Funct. Anal. 263 (2012), no. 11, 3492-3563. MR 2984074

[How85] Roger Howe, Dual pairs in physics: harmonic oscillators, photons, electrons, and singletons, Applications of group theory in physics and mathematical physics (Chicago, 1982), Lectures in Appl. Math., vol. 21, Amer. Math. Soc., Providence, RI, 1985, pp. 179-207. MR 789290 (86i:22036)

[How89a] Remarks on classical invariant theory, Trans. Amer. Math. Soc. 313 (1989), no. 2, 539-570. MR 986027 (90h:22015a)

[How89b] _ Transcending classical invariant theory, J. Amer. Math. Soc. 2 (1989), no. 3, 535552. MR 985172 (90k:22016)

[HU91] R. Howe and T. Umeda, The Capelli identity, the double commutant theorem, and multiplicity-free actions, Math. Ann. 290 (1991), 565-619.

[KR71] B. Kostant and S. Rallis, Orbits and representations associated with symmetric spaces, Amer. J. Math. 93 (1971), 753-809. MR 0311837 (47 \#399)

[KV78] M. Kashiwara and M. Vergne, On the Segal-Shale-Weil representations and harmonic polynomials, Invent. Math. 44 (1978), no. 1, 1-47. MR 0463359 (57 \#3311)

[KV95] A. W. Knapp and D. A. Vogan, Cohomological induction and unitary representations, Princeton Mathematical Series, vol. 45, Princeton Univ. Press, 1995.

[Woo91] N. Woodhouse, Geometric quantization, Oxford Univ. Press, 1991.

University Education Center, Tottori University, 4-101, Koyama-Minami, TotTORI, 680-8550, JAPAN

E-mail address: thashi@uec.tottori-u.ac.jp 